\title{
Avifauna del Parque Nacional Natural Selva de Florencia (Samaná, Caldas, Colombia): nuevos registros y ampliaciones de distribución
}

\author{
Avifauna of the Selva de Florencia National Natural Park \\ (Samaná, Caldas, Colombia): new records and distribution \\ extensions
}

\author{
Daniela Gómez $^{(i)}$, Kelly Orozco ${ }^{(i)}$, Felipe Cardona ${ }^{(i)}$, Milton Pineda ${ }^{(i)}$, Mary Luz Beboya ${ }^{\left({ }^{\circ}\right.}$, \\ David Ocampo (i)
}

\section{Resumen}

El Parque Nacional Natural Selva de Florencia es considerado uno de los últimos fragmentos de bosque pluvial andino de la cordillera Central de Colombia. Con el propósito de actualizar el estado del conocimiento de la avifauna del Parque recopilamos información obtenida mediante monitoreos realizados desde 2012 y registros de dos expediciones realizadas entre 2017 y 2018. Registramos 357 especies, de las cuales 11 son endémicas y 20 tienen alguna categoría de amenaza. Reportamos 18 nuevas especies para el departamento, con ampliaciones en el rango de su distribución para 12 de ellas. El contacto de ensamblajes de especies de bosques montanos con los de especies de influencia del valle del Magdalena medio, sumado al buen estado de conservación y regeneración de los ecosistemas, en gran medida producto del restringido acceso generado por el conflicto armado, podrían explicar la gran riqueza de especies de aves en la zona $(\approx 20 \%$ de las especies de Colombia en menos del $0.01 \%$ del territorio). Esta nueva información contribuye al conocimiento de la distribución de la avifauna en los Andes Centrales y reafirman al PNN Selva de Florencia como una zona prioritaria para el estudio y la conservación de la biodiversidad.

Palabras clave. Andes. Aves. Cordillera Central. Parques Nacionales Naturales de Colombia.

\begin{abstract}
The Selva de Florencia National Natural Park is one of the last fragments of the Andean rain forest in the Central Cordillera of Colombia. With the purpose of updating the state of knowledge of the park's avifauna, we collected information obtained through monitoring carried out by park officials since 2012 and records of two expeditions made between 2017 and 2018. We recorded 357 species, of which 11 are endemic and 20 are in some threat category. We report 18 new records for the department and extensions in the distribution range for 12 of these species. The contact between the assemblies of montane forest species with those from the Middle Magdalena valley, added to the good state of conservation of the park and the regeneration of ecosystems, largely due to the restricted access generated by the armed conflict, could explain the great richness of bird species in the area $(\approx 20 \%$ of the Colombian species in less than $0.01 \%$ of its territory). This new information contributes to the knowledge of the bird distribution of in the Central Andes and stresses the importance of the Selva de Florencia National Natural Park as a priority area for the study and conservation of biodiversity.
\end{abstract}

Key word. Andes. Birds. Cordillera Central. National Natural Parks of Colombia. 


\section{Introducción}

Debido a la gran variedad de hábitats y ecosistemas, la cordillera Central, en el norte de los Andes, se ha identificado como un centro de biodiversidad importante, promovida por cambios en la elevación y la heterogeneidad topográfica y climática (Terborgh, 1971; Kattan et al., 2006). En esta zona se han identificado y delimitado áreas estratégicas para la conservación in situ, donde hasta el momento se han declarado seis Parques Nacionales, a lo largo de gradientes de elevación, desde las tierras bajas en los valles interandinos hasta zonas de gran elevación (Parques Nacionales Naturales de Colombia, 2019). Una de estas áreas de gran importancia es el Parque Nacional Natural (PNN) Selva de Florencia, considerado como el último fragmento de bosque pluvial andino del cinturón cafetero en la cordillera Central, caracterizado por poseer una enorme riqueza y endemismo de fauna y flora (Ballesteros et al., 2017).

El objetivo principal del Parque es conservar los biomas de bosque húmedo subandino y bosque húmedo altoandino, con el fin de preservar el hábitat de especies endémicas y amenazadas y de contribuir al proceso de conectividad en la ecorregión del Eje Cafetero, el Magdalena medio caldense y el oriente antioqueño (Herrera et al., 2016; Ballesteros et al., 2017). Algunos estudios en el parque evidencian una gran diversidad biológica, en los que se reconocen hasta ahora 75 especies de anfibios (17 endémicas y 31 amenazados) y 75 de mamíferos, siendo de vital importancia la presencia de primates amenazados (Ballesteros et al., 2017). Para aves se han reportado cerca de 300 especies, otorgándole al parque el título de AICA (Área Importante para la Conservación de las Aves), debido a la alta concentración de aves endémicas y amenazadas a nivel global (Franco \& Bravo, 2005). Históricamente el parque fue corredor de paso de grupos armados, dejando zonas inaccesibles en buena parte del área protegida. Sin embargo, gracias a los acuerdos de paz y a las nuevas posibilidades de acceso, se han realizado recientemente varias visitas y una expedición, liderada por investigadores de la Universidad de Caldas, que abarcó todos los grupos taxonómicos, llamada Caldas Expedición Siglo XXI.

Teniendo en cuenta la importancia estratégica que tiene el parque para la avifauna de la región y el país, y en vista de que existe información fragmentada sin publicar sobre el parque y nuevas iniciativas de documentación de su biodiversidad, recopilamos la información de inventarios con registros históricos y realizamos dos expediciones en algunos sectores del área protegida. El propósito de esta contribución es actualizar el estado de conocimiento de la avifauna de este territorio poco explorado y registrar y confirmar la presencia de especies, integrando diferentes actores del departamento en procesos dinámicos de investigación y documentación de la biodiversidad.

\section{Materiales y métodos}

Área de estudio. El PNN Selva de Florencia está ubicado sobre la vertiente oriental de la cordillera Central en el departamento de Caldas (Figura 1), en zona limítrofe de los municipios de Samaná y Pensilvania, en jurisdicción de los corregimientos de Florencia y Encimadas (Samaná) y Pueblo Nuevo (Pensilvania). Tiene una extensión de 10019 ha, con influencia de las cuencas hidrográficas de los ríos La Miel y Samaná Sur, afluentes del río Magdalena (Ballesteros et al., 2017). Presenta un gradiente de elevación entre 850 y 2400 m s.n.m. una precipitación media anual de $6270 \mathrm{~mm}$ (Ballesteros et al., 2017) y se encuentra categorizado como Zonobioma húmedo tropical, Orobioma andino y Orobioma subandino (Rodríguez et al., 2006; IDEAM, 2007).

El listado de especies representa registros de tres fuentes de información principales: 1) censos en 212 puntos fijos realizados por funcionarios de parques, cada uno con tres repeticiones, los cuales suman $106 \mathrm{~h}$ de observación, más los recorridos libres en cada sendero; 2 ) muestreos de equipo de biólogos de la Universidad de Caldas; y 3) una expedición y visitas cortas de un equipo mixto entre biólogos, estudiantes y observadores de aves involucrados en procesos de ciencia participativa. En estos últimos obtuvimos un esfuerzo de $26 \mathrm{~h}$ de observación en recorridos libres y un esfuerzo con redes de niebla de 272.15 m.red-hora ${ }^{-1}$.

El primer equipo, conformado por tres funcionarios del parque con experiencia en observación de aves, realiza visitas dos o tres veces al mes a cada ruta de los distintos sectores del parque. Cada ruta es realizada por un observador que hace el recorrido de aproximadamente 4 h de observación; estas son:

1 Sector Samaná (Rutas S1, S2, S3, S4, S5, S6). Se encuentra entre los 1200 y 2000 m s.n.m. Pertenece a zonas de bosque húmedo subandino, con presencia de mosaicos de cultivos de café, pastos, zonas de rastrojos bajas y altas, además de áreas de bosque muy conservado principalmente en la ruta S6 (Figuras 2A y B). 


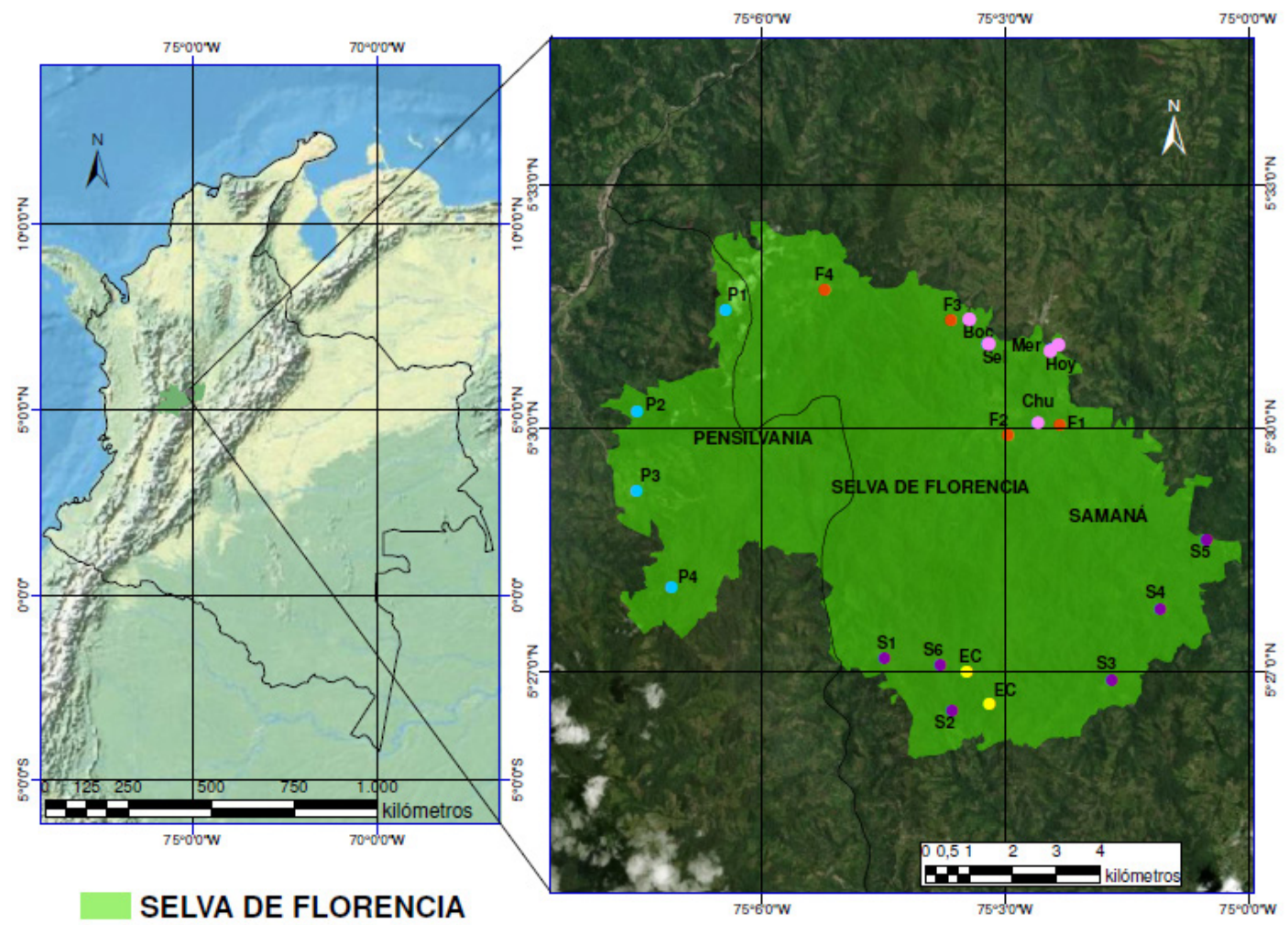

Figura 1. Ubicación Parque Nacional Natural Selva de Florencia, Caldas, Colombia. Puntos de muestreo: azul, sector Pensilvania; rojo, sector Florencia; violeta, sector Samaná; amarillo, expedición Aves en Caldas (EC); rosado, Caldas Expedición Siglo XXI.

2 Sector Florencia (Rutas F1, F2, F3 y F4). Se encuentra entre los 1050 y 1700 m s.n.m. en zonas de bosque húmedo subandino, con presencia de bosques secundarios y cultivos de café y caña. Presenta algunos pastizales y áreas en recuperación natural. Las zonas con abundante agua y bosques primarios están localizadas en las rutas F1 y F4 (Figuras 2C y D).

3 Sector Pensilvania (Rutas P1, P2, P3, P4). Se encuentra entre los 1000 y 2350 m s.n.m. con presencia de áreas abiertas entre bosques secundarios, zonas agrícolas y plantaciones de pino pátula y ciprés, considerándose así el sector con mayor transformación del parque. Las rutas $\mathrm{P} 3$ y $\mathrm{P} 4$ poseen bosques andinos muy conservados y zonas de regeneración natural (Figuras 2E y F).

El segundo equipo estuvo integrado por seis investigadores divididos en dos grupos, con el fin de implementar metodologías de muestreo complementarias (censos, redes de niebla), en localidades pertenecientes a la subcuenca del río San Antonio y el sector Las Bocatomas, ubicados en la zona del corregimiento de
Florencia. El primer muestreo se llevó a cabo entre el 19 y 21 de octubre de 2017 en la microcuenca La Selva, en zona de bosque húmedo subandino y altoandino, el segundo muestreo se realizó entre el 20 y 24 de febrero de 2018, en los sectores Las Mercedes y Las Bocatomas (bosque húmedo subandino) y el tercero en la microcuenca Chupaderos y el sector El Hoyo (bosque subandino) entre el 20 y 25 de abril de 2018.

Finalmente, el tercer equipo, liderado por biólogos y con el apoyo de funcionarios y observadores de aves locales, estuvo en zonas correspondientes a bosque subandino en el sendero S6 del Parque, conocido como Boquerón-Yarumal (Sector Samaná) el 20 de junio de 2018.

\section{Toma de datos.}

Debido a la heterogeneidad en las intensidades de muestreo e integrantes de equipos, se dificulta un análisis comparativo; por ello nos limitamos a presentar un listado de especies registradas en el parque. En todos los casos, para la detección realizamos 

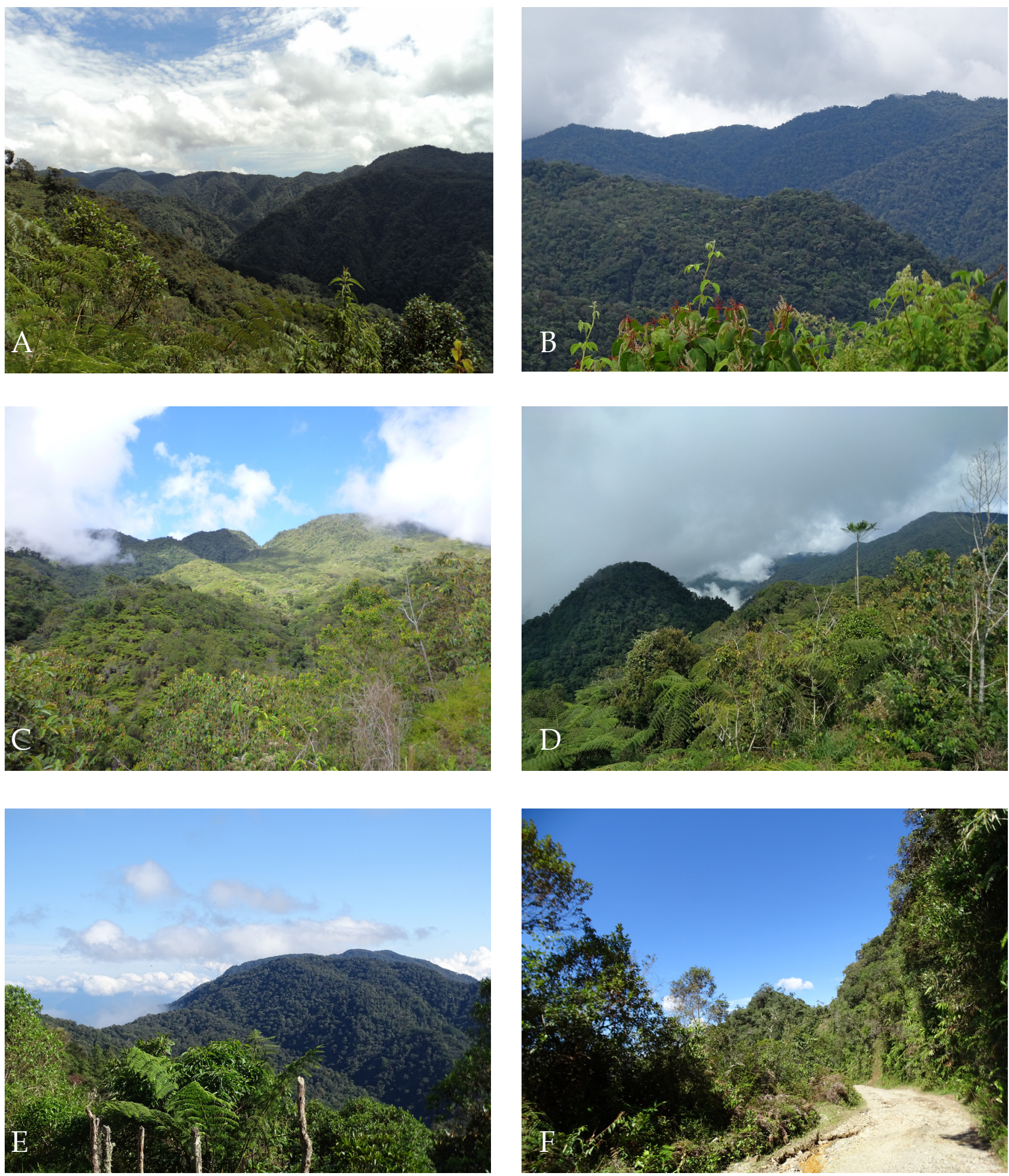

Figura 2. Cobertura vegetal asociada a los distritos de manejo del Parque Nacional Natural Selva de Florencia, Caldas, Colombia. A y B, sector Samaná; C y D, sector Florencia; E y F, sector Pensilvania. Fotografías: Felipe Cardona. 
metodologías complementarias como recorridos libres, puntos de conteo, capturas con redes de niebla, grabación de vocalizaciones y algunas estaciones con cámaras trampa marca Bushnell. Los recorridos de observación y puntos de conteo fueron realizados en las horas de mayor actividad de las aves, entre las 6:00 y 10:00 h y entre 15:00 y 18:00 h. Simultáneamente realizábamos grabación de vocalizaciones con una Grabadora H4n 4-Channel Handy adaptada a un micrófono ME66/K6 Sennheiser. Para las observaciones utilizamos binoculares $8 \times 40$ y 10x50, y para el registro fotográfico cámaras compactas.

Durante las expediciones tuvimos sesiones de captura de aves utilizando redes de niebla $(12 \times 2.5 \mathrm{~m})$, que fueron ubicadas en coberturas de interés (bosque de galería, vegetación arbustiva o zonas de transición) siguiendo a Ralph et al. (1996) y Villarreal et al. (2006). Para la identificación usamos las guías de campo de Hilty \& Brown (2001) y Ayerbe-Quiñones (2018). Para la nomenclatura taxonómica seguimos la propuesta de la SACC (Remsen et al., 2020; en: https:/ / bit.ly/2PAPYFG); identificamos endemismos (Avendaño et al., 2017) y estatus de conservación según la IUCN 2019 (https://www.iucnredlist.org/search) y el Libro rojo de las aves de Colombia (Renjifo et al., 2014). Adicionalmente, revisamos la colección ornitológica del Museo de Historia Natural de la Universidad de Caldas (MHN-UC) y la Colección de Aves del Instituto Humboldt (IAvH-A).

Para consolidar el listado, realizamos curaduría de toda la información de los monitoreos realizados desde el año 2012, basados en especímenes de colecciones biológicas y mapas revisados de la distribución de las especies. La información de los monitoreos desde el año 2017 se ha sistematizado en la plataforma SULA de Parques Nacionales. La evidencia de los registros nuevos, por parte de funcionarios del parque, se revisó con registros fotográficos y fueron validados por los ornitólogos del equipo. Las observaciones fueron ingresadas en la plataforma de libre acceso eBird desde 2017. Los registros novedosos fueron elegidos mediante el análisis de distribución de potenciales especies encontradas durante los muestreos. Todos estos registros corresponden a nuevos registros confirmados para el departamento, lo que implica una ampliación de la distribución de varios $\mathrm{km}$ (ver resultados) al sur de la vertiente oriental de la cordillera Central, y solo fueron incluidos si contaban con evidencia física (fotografía) de su presencia en el parque.

\section{Resultados}

Consolidamos un listado de 357 especies de aves (Anexo 1). Las familias más representativas fueron Thraupidae (56 spp.), Tyrannidae (42), Trochilidae (29) y Furnariidae (23), que aportan el 43\% del total de la riqueza de especies; reportamos además 28 especies migratorias boreales pertenecientes a nueve familias. Con las redes niebla capturamos 91 individuos de 38 especies; las especies de mayor frecuencia de capturas fueron Mionectes olivaceus, con cinco individuos, y Eutoxeres aquila, Basileuterus tristriatus y Haplophaedia aureliae, con tres individuos cada una.

Reportamos 18 especies en alguna categoría de amenaza según la IUCN, seis de ellas a nivel nacional (Anexo 1), y confirmamos la presencia de 18 especies que se encontraban como probables registros para el departamento (Corpocaldas \& Asociación Calidris, 2010): Odontophorus erythrops, Patagioenas speciosa, Discosura conversii, Lophostrix cristata, Micromonacha lanceolata, Pyrilia pyrilia, Touit dilectissimus, Grallaricula flavirostris, Formicarius rufipectus, Sclerurus mexicanus, Pseudotriccus pelzelni, Knipolegus poecilurus, Pipreola aureopectus, Ampelioides tschudii. Chlorochrysa nitidissima, Iridosornis porphyrocephalus, Arremon castaneiceps y Saltator grossus. Entre estas, para 12 es el registro más al sur de su distribución conocida en la vertiente oriental de la cordillera Central:

Odontophorus erythrops, perdiz collareja. Esta especie habita el sotobosque de selvas y bosques húmedos, con mayor probabilidad de detección por vocalizaciones (Restall et al., 2007). De hecho, se pudo registrar solo en cámaras trampa instaladas por funcionarios del Parque en el año 2016 (Figura 3), en las veredas la Cabaña, del corregimiento de Florencia, en Samaná. $\left(05^{\circ} 29^{\prime} 39,1^{\prime \prime} \mathrm{N}-75^{\circ} 01^{\prime} 27,9^{\prime \prime}\right.$ O. 1458 m s.n.m.). En el Museo de Historia Natural de la Universidad de Caldas se tiene respaldo de un espécimen (MHN-UC-25) de la vereda La Abundancia, sector Florencia. Su distribución abarca la vertiente occidental de la cordillera Occidental y el norte de la cordillera Central, por debajo de los $1700 \mathrm{~m}$ de elevación (Ayerbe-Quiñones, 2018), en donde se ha reportado en Antioquia en la Reserva del Arrierito Antioqueño (Cuervo et al. 2008b) y en los límites de este departamento con el sur de Bolívar (Serranía de San Lucas), sector de Santa Cecilia (Donegan, 2012). 


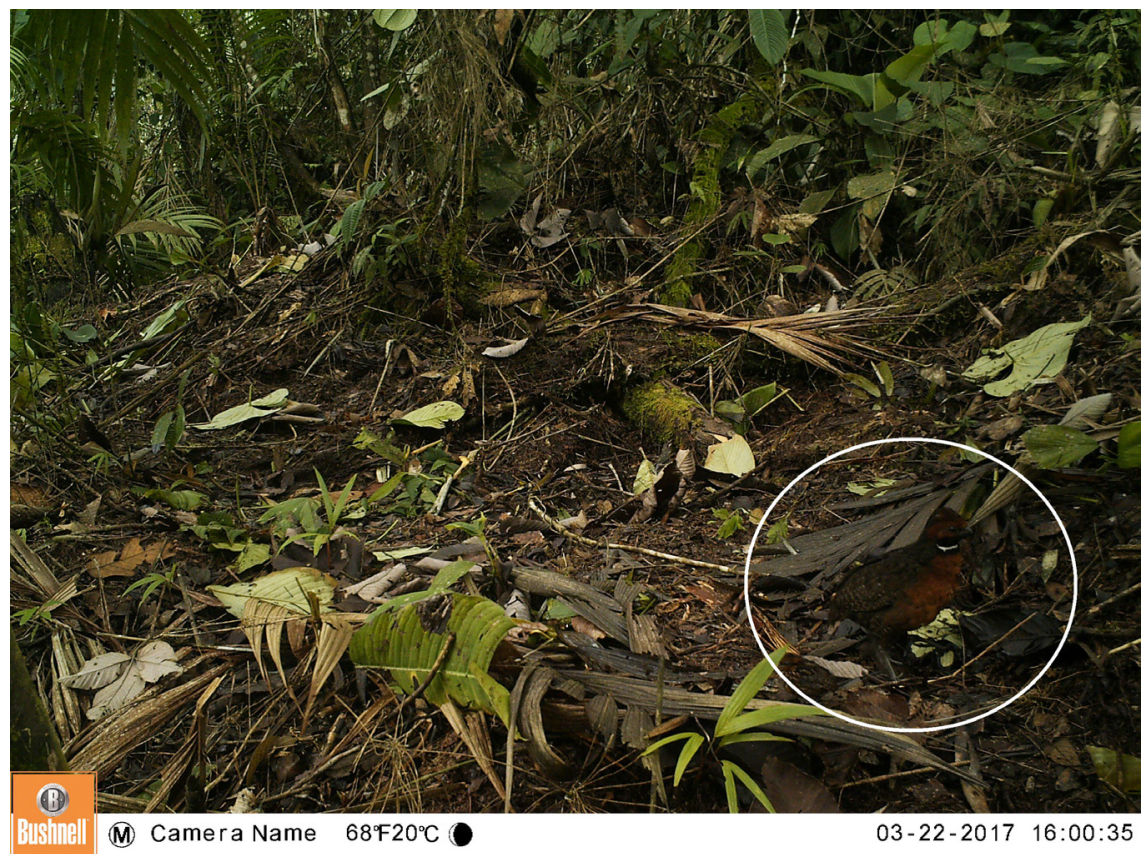

Figura 3. Fotografía de cámara trampa de la perdiz collareja (Odontophorus erythrops) en el Parque Nacional Natural Selva de Florencia, Caldas, Colombia. Fotografías: Archivo Parques Nacionales Naturales.

Lophostrix cristata, búho crestado. El 30 de julio del 2018 fue fotografiado un adulto en zona de bosque primario en la Vereda El Quindío (Figura 4), Sector de manejo Samaná $\left(5^{\circ} 27^{\prime} 4.3^{\prime \prime} \mathrm{N} 75^{\circ} 02^{\prime} 33.9^{\prime \prime} \mathrm{O} .1630 \mathrm{~m}\right.$ s.n.m.). No se tenían reportes de esta especie para el departamento (Corpocaldas \& Asociación Calidris, 2010) y poco se sabe sobre su ecología y comportamiento en Colombia (Chaparro-Herrera et al., 2015). Se tiene reporte documentado en la Serranía de las Quinchas (Stiles \& Bohórquez, 2000) y registros puntuales para la cordillera Central en La Reserva Cañón de Río Claro (Antioquia) y La Virginia, Tolima según eBird 2019 y Sergio Chaparro-Herrera (obs. pers.). La distribución de esta especie comprende la cordillera Occidental, base norte de los Andes hasta el valle medio del Magdalena y en el sur incluye la región de piedemonte, abarcando Caquetá, Putumayo y Amazonas, bajo 1000 m de elevación (Ayerbe-Quiñones, 2018). Con este registro confirmamos la presencia de la especie en la cordillera Central, a $600 \mathrm{~m}$ por encima del límite máximo de su elevación reportada.

Pyrilia pyrilia, cotorra cariamarilla. Un individuo fue fotografiado el 3 de noviembre de 2017 en el sector Florencia conocido como La Vega (ruta F1). Esta especie habita selvas húmedas y bosques montanos en dosel y bordes, donde se moviliza usualmente en grupos pequeños (Restall et al., 2007). Su distribución abarca parte de la vertiente occidental de la cordillera Occidental, base norte de los Andes hacia el Magdalena medio y norte de la vertiente oriental en la cordillera Oriental, por debajo de $1700 \mathrm{~m}$ de elevación (Ayerbe-Quiñones, 2018). Ha sido registrada por Cuervo et al. (2008a) en Amalfi y Anorí (Antioquia), y en el Cañón del Rio Claro, Puerto Triunfo (Antioquia) (D. Ocampo obs. pers.), por lo que este reporte representaría una ampliación del rango de distribución hacia la región centro-sur de la cordillera Central.

Touit dilectissimus, periquito alirrojo. Es considerada rara o poco común (Rodríguez-Mahecha \& Hernández- Camacho, 2002), con distribución a lo largo de la cordillera Occidental y en la región más al norte de la Central y Oriental entre 500 y 2300 m de elevación (Ayerbe-Quiñones, 2018). Fue observada el 15 de agosto de 2017 en el sector Samaná, vereda El Quindío (ruta S3). Previamente registrada por Cuervo et al. (2008b) y Olaciregui \& Urueña (2011) en Amalfi y Anorí (Antioquia).

Discosura conversii, cola de lira verde. Este colibrí habita estratos altos de bosques, selvas húmedas, bordes y claros, donde se alimenta de néctar e insectos (Restall et al 2007). El 31 de agosto de 2018, en el sector Diamante,

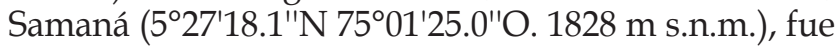
observada una hembra en disputa por territorio ante un 
individuo de Thalurania colombica, en una zona de rastrojo y regeneración natural. Su distribución abarca la vertiente occidental de la cordillera Occidental y la base norte de los Andes hacia el valle del Magdalena medio, entre 100 y 1200 m de elevación (Ayerbe-Quiñones, 2018); este registro fue a más de $600 \mathrm{~m}$ por encima de la elevación conocida para la especie.

Micromonacha lanceolata, bigotudo lanceolado. Habita estratos medios al interior de bosques y vegetación secundaria en zonas húmedas; en Colombia se encuentra por debajo de $2000 \mathrm{~m}$ de elevación en la cordillera Occidental, en el departamento de Antioquia, y al oriente de los Andes desde la Serranía de la Macarena, Nariño, Putumayo y Caquetá (Ayerbe-Quiñones, 2018). Fue registrada y fotografiada (Figura 5A) en 2016 en las veredas San Lucas, corregimiento de Florencia (Samaná) y en 2018 en Yarumalito, sector de manejo de Samaná, donde además se registró un nido con dos polluelos $\left(05^{\circ} 26^{\prime} 42.5^{\prime \prime} \mathrm{N}-7^{\circ} 03^{\prime} 55.2^{\prime \prime} \mathrm{O} .1964 \mathrm{~m}\right.$ s.n.m.). La especie ha sido reportada previamente en Antioquia, municipio de Anorí (Cuervo et al., 2008b), por lo que nuestro registro sería el segundo publicado para la cordillera Central, $170 \mathrm{~km}$ más al sur, lo que sugiere una distribución continua entre los fragmentos de bosque de la vertiente oriental de la cordillera Central. Según Cuervo et al. (2008b), es una especie poco representada en las colecciones y por esta razón existe aún incertidumbre sobre el conocimiento taxonómico y las afinidades filogenéticas entre poblaciones para el país.

Grallaricula flavirostris, tororoi piquigualdo. En Colombia se encuentra entre los 500 y 2200 m, a lo largo de la vertiente oriental de la cordillera Oriental, la vertiente occidental de la cordillera Occidental y en los extremos norte y sur de la cordillera Central (Ayerbe-Quiñones, 2018). En junio del 2018 observamos y fotografiamos un individuo forrajeando entre el sotobosque en el sendero S6 en el sector la CuchillaBoquerón (Figura 5B), vereda Yarumal, Samaná (05²6' $15.17^{\prime \prime} \mathrm{N}-75^{\circ} 02^{\prime} 56.94^{\prime \prime} \mathrm{O} .1178 \mathrm{~m}$ s.n.m.). No existían indicios de la presencia de esta especie en el departamento de Caldas; sin embargo, se han documentado observaciones de comportamiento reproductivo (Delgado, 2002) y registros para la cordillera Central en Antioquia, principalmente al norte en Anorí y Amalfí (Peña, 1998; Cuervo et al., 2008b). Por lo anterior, este reporte podría sugerir una ampliación del rango de distribución de más de $150 \mathrm{~km}$ al sur, a lo largo de la cordillera Central. El género Grallaricula es considerado como uno de los grupos de aves menos conocido en Surámerica (DelgadoV, 2002); según Cuervo et al (2008b) existen vacíos de información sobre la taxonomía de esta especie, ya que algunos especímenes evidencian plasticidad fenotípica, que sumado al estado de amenaza (NT), según IUCN (2019), alerta sobre la necesidad de aumentar en el conocimiento sobre su biología y distribución.

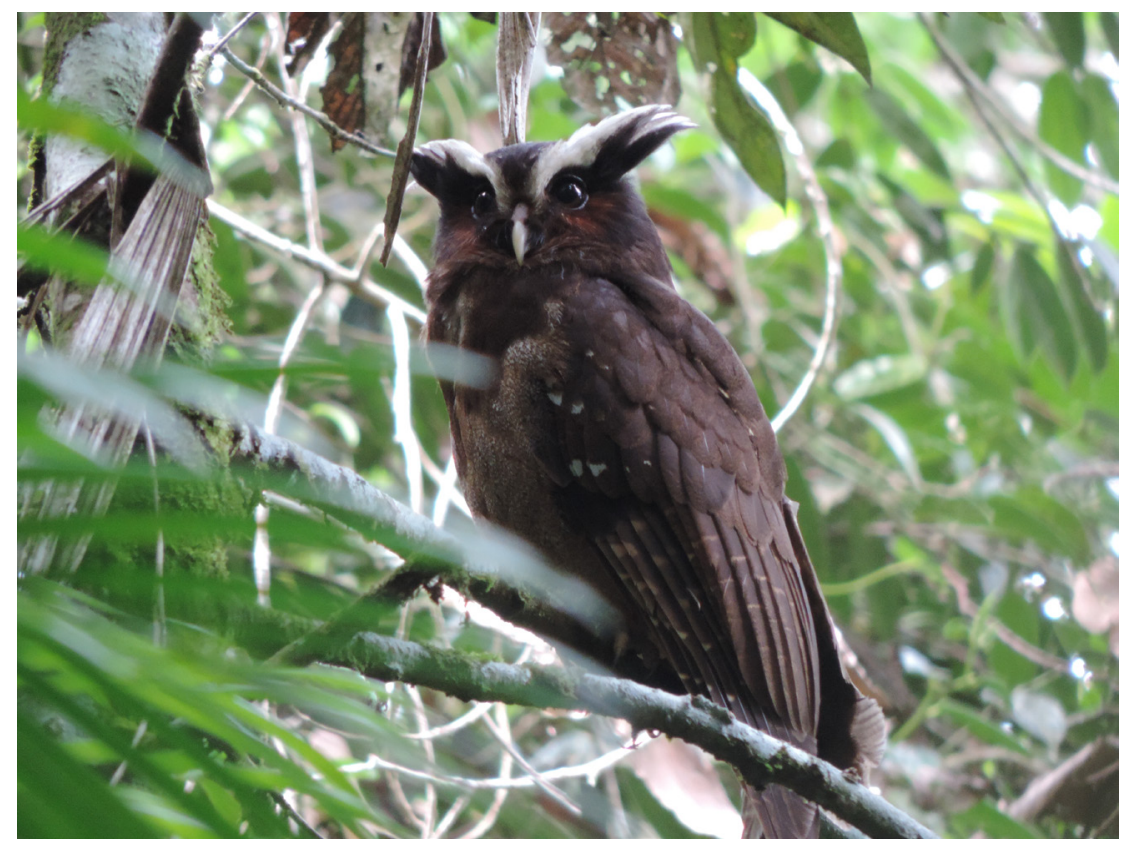

Figura 4. Fotografía del búho crestado (Lophostrix cristata) en la Vereda El Quindío (Sector Samaná), del Parque Nacional Natural Selva de Florencia, Caldas, Colombia. Fotografía: Milton Pineda. 
Pipreola aureopectus, frutero pechidorado. Esta especie habita en bosques húmedos montanos, donde forrajea en parejas o bandadas mixtas en los estratos medios y altos del bosque (Hilty \& Brown, 2001; Restall et al., 2007). Fue observada en 2016 en la vereda de San Francisco (Figura 5C), corregimiento de Pueblo Nuevo, Samaná (05030'58.86"N-7502'24.48"O 1581 m s.n.m) y en 2018 en el sendero S6 en el sector la Cuchilla-Boquerón, vereda Yarumal, Samaná $\left(05^{\circ} 26^{\prime} 15.17^{\prime \prime} \mathrm{N}-75^{\circ} 02 ' 56.94^{\prime \prime} \mathrm{O}\right.$. $1178 \mathrm{~m}$ s.n.m.). Son escasos los registros de esta especie en la cordillera Central: Cuadros (1988) la reporta en la subregión norte de Antioquia (Carolina del Príncipe) y Corpocaldas y Universidad de Caldas (2001) también reportan la especie en el plan de manejo del PNN Selva de Florencia; sin embargo, no había evidencia corroborada y actual de su presencia en la zona.

Ampelioides tschudii, frutero escamado: Se observó y fotografió un macho el 17 de octubre de 2019 en el predio La frontera (Figura 5D), vereda Divisa (0530'26.6"N 750 $7^{\prime} 07.5^{\prime \prime} \mathrm{O} .1561 \mathrm{~m}$ s.n.m.), corregimiento Pueblo Nuevo (municipio de Pensilvania). El registro se obtuvo mientras se realizaba monitoreo de Ateles hybridus en zonas de bosque secundario. Este registro es el primero de la especie en el departamento, con el cual confirmamos su ampliación de distribución a 165 $\mathrm{km}$ al sur de la cordillera Central de acuerdo al reporte de Castaño- Hernández \& Calderón-Franco (2012) en La Reserva del Arrierito antioqueño en Anorí.

Iridosornis porphyrocephalus, musguerito gargantilla. Es común observar esta especie forrajeando en parejas y bandadas mixtas en estratos bajos y medios de bosques altoandinos (Restall et al., 2007). En los recorridos del parque se observó un individuo (Figura 5E) en 2018 en la vereda Yarumal del corregimiento de Encimadas, Samaná $\left(05^{\circ} 27^{\prime} 13.98^{\prime \prime}\right.$ N-7502' 59.94"O. 1790 m s.n.m.). En la cordillera Central se conocen reportes en Antioquia en Carolína del Princípe (Cuadros, 1988), Amalfi y Anorí (Cuervo et al., 2008b). El registro realizado por Castaño-Villa \& Patiño-Zábala (2008) en Santa Helena (Medellín) a $97 \mathrm{~km}$ al norte, sería el más cercano a la zona en donde registramos la especie.

Saltator grossus, picogordo pizarra. Esta especie fue reportada mediante observación directa en 2018 (Figura 5F), con un único registro en el sector Los Planes, vereda El Quindío, en el municipio de Samaná (05'26'53.2"N-7501'41.7"O. 1478 m s.n.m.). Los registros se presentaron en ecosistemas que anteriormente tuvieron algún grado de intervención y que hoy ya se encuentran recuperados. Los reportes previos en la cordillera Central han sido en el municipio de Pereira (Londoño-Betancourth, 2011) y en ecosistema de bosque seco tropical en Chucuní, municipio de Ibagué, Tolima (Lozada \& Molina, 2011).

Arremon castaneiceps, pinzón oliva. Se distribuye entre 700 y 2200 m en la vertiente Occidental de la cordillera Occidental y en la cordillera Oriental al sur en los departamentos de Nariño y Caquetá (Ayerbe-Quiñones, 2018). Esta especie había sido sugerida para la zona por Corpocaldas \& Asociación Calidris (2010), pero no existía evidencia confirmada de su presencia. Capturamos un individuo el 23 de febrero de 2018 en el Sector Las Bocatomas, Florencia $\left(05^{\circ} 31^{\prime} 16.1^{\prime \prime} \mathrm{N} 75^{\circ} 03^{\prime} 29.5^{\prime \prime} \mathrm{O}\right.$. 1723 m s.n.m.). El individuo, al parecer juvenil por el grado intermedio de osificación craneal, no presentaba indicios de muda. Este registro sería el primero documentado, de nuestro conocimiento, para la especie en la cordillera Central (Figura 5G). Sin embargo, reportes recientes en eBird de otras localidades de Caldas y en Quindío, sugieren que podría estar aún más al sur en la cordillera y que seguramente es más común de lo que parece.

El 20, 21 y 22 de febrero de 2018, se observó una pareja de Spizaetus ornatus anidando (Figura 6). Esta evidencia es de vital importancia, ya que en Colombia se conocen pocos registros de nidos de esta especie (Sebastián Restrepo com. pers.), la cual es un poderoso depredador de los bosques húmedos bien conservados del neotrópico (Márquez et al., 2005; Restall et al., 2007). Su presencia, y en general la diversidad de aves rapaces, son indicadores de bosques con alto grado de conservación en el área protegida.

Durante la búsqueda de evidencia de especies en colecciones biológicas provenientes de expediciones de aves en el parque, encontramos especímenes de: Odontophorus erythrops (MHN-UC-25) y Mionectes olivaceus (MHN-UC-147), en el Museo de Historia Natural de la Universidad de Caldas, y especímenes de Myiarchus cephalotes (IAvH-A 06442, IAvH-A 06443), Adelomyia melanogeys (IAvH-A 06446), Heliodoxa rubinoides (IAvH-A 06444), y Pseudotriccus pelzelni (IAvH-A 06445) en la Colección de Aves del Instituto Humboldt. No existe ninguna grabación de vocalizaciones de cantos de aves en el parque en repositorios nacionales, como la Colección de Sonidos Ambientales del Instituto Humboldt (IAvH-CSA). 


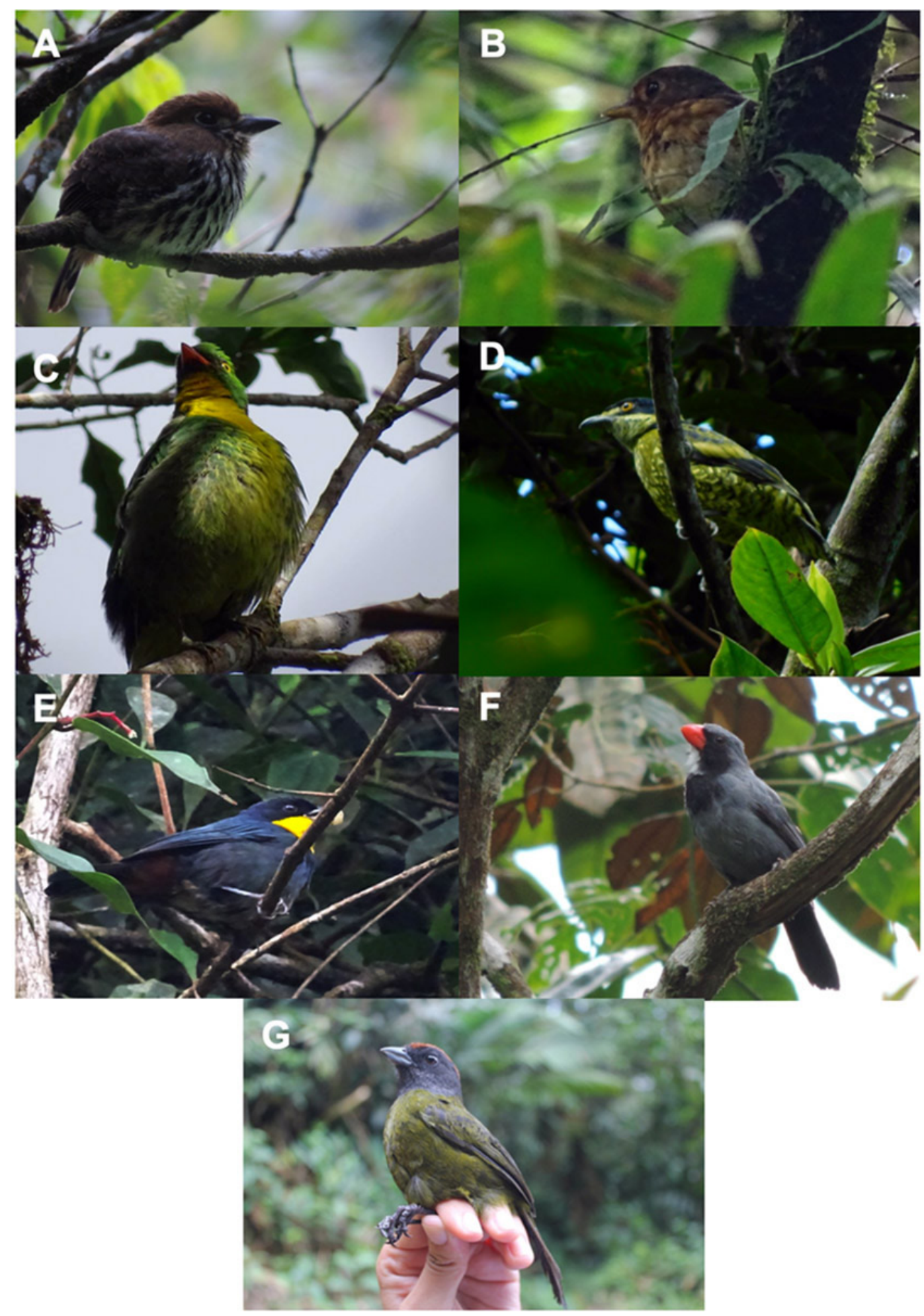

Figura 5. Algunas aves del del Parque Nacional Natural Selva de Florencia, Caldas, Colombia: A, bigotudo lanceolado (Micromonacha lanceolata); B, tororoi piquigualdo (Grallaricula flavirostris); C, frutero pechidorado (Pipreola aureopectus); D, frutero escamado (Ampelioides tschudii) E, musguerito gargantilla (Iridosornis porphyrocephalus); F, picogordo pizarra (Saltator grossus); G, pinzón oliva (Arremon castaneiceps). Fotografías: Archivo Parques Nacionales Naturales. A y E, Milton Pineda Duque; B, C y D, Felipe Cardona Toro; F, Santiago Cardona Toro; G, Alejandra María Patiño Gallego. 

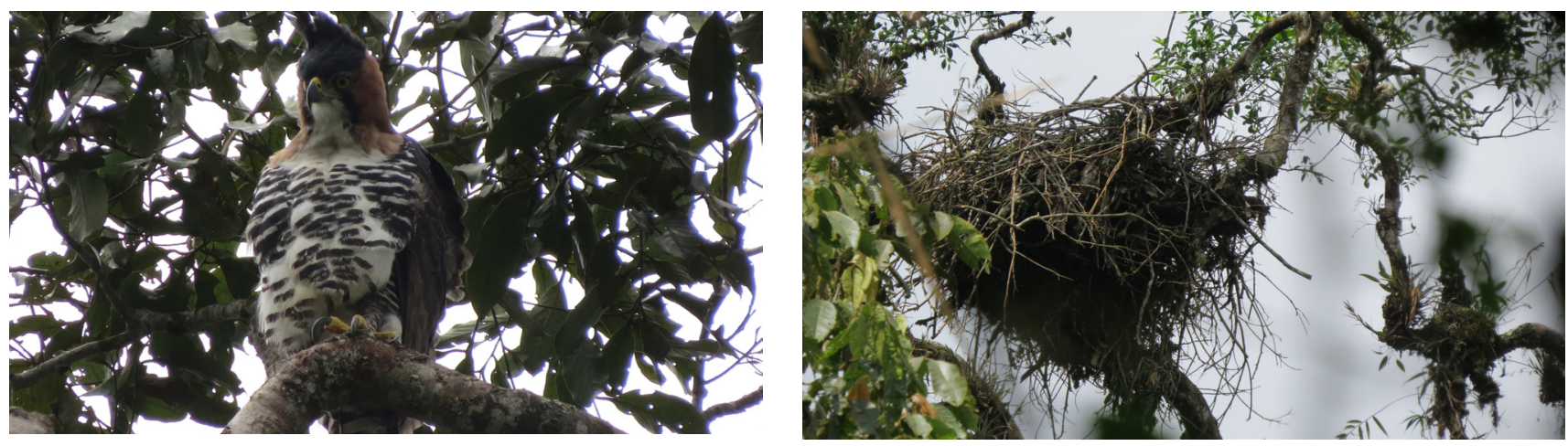

Figura 6. Spizaetus ornatus y su nido, en el del Parque Nacional Natural Selva de Florencia, Caldas, Colombia. Fotografía: Nicolás Botero Henao.

\section{Discusión}

De las 300 especies registradas en el parque antes de nuestro estudio, se reportaron cinco en categoría de amenaza a nivel nacional. En Peligro: Atlapetes flaviceps, Scytalopus stilesi, Vulnerables: Hypopyrrhus pyrohypogaster, Capito hypoleucus y Chlorochrysa nitidissima. Por otra parte, solo se tenía registro de 14 especies migratorias (Parques Nacionales Naturales de Colombia, 2019). De esta manera, las especies encontradas complementan los inventarios reportados por Corpocaldas y Universidad de Caldas (2001), Castellanos et al. (2003) y Paiba-Alzate et al. (2010), permitiendo ampliar incluso el número de especies para el departamento. En estos inventarios iniciales, Castellanos et al. (2003) reportan en el parque especies amazónicas, como Trogon comptus y Ciccaba huhula, las cuales no registramos en este estudio, y consideramos que probablemente fueron identificaciones erróneas.

La efectividad de las acciones de conservación en estas áreas protegidas se ha considerado limitada, en parte porque el conocimiento base dentro de cada área es muy escaso y en muchos casos es basada en información secundaria de trabajos antiguos (Linares-Romero et al., 2020). Es por esto que esta nueva información se convierte en una base importante para generar alianzas institucionales, académicas y con las comunidades locales que permitan desarrollar investigación ecológica, de manejo del paisaje y de educación para el seguimiento de las especies focales y su conservación.

En Colombia, la mayoría de adiciones y ampliaciones de distribución de las aves provienen de regiones al occidente de la cordillera Oriental (trans-Andes) y zonas costeras del país (Avendaño et al., 2017). Nuestros hallazgos coinciden con otros patrones de ampliación de distribución de la avifauna para la cordillera Central, pues los registros corresponden a especies de estribaciones pluviales del Chocó en la cordillera Occidental (Cuervo et al., 2008b). Tal es el caso de Grallaricula flavirostris y Arremon castaneiceps, de las cuales no se tenía evidencia para el departamento. La Provincia Biogeográfica de Chocó-Magdalena, a la cual pertenece el parque, es considerada una de las zonas con mayor precipitación del país (Ballesteros et al., 2007), por lo que esta configuración ambiental, sumada al estado de conservación de la zona, puede explicar la presencia de algunas especies de la vertiente occidental de la cordillera Occidental.

Por otro lado, la documentación de la ornitofauna en los parques nacionales sigue siendo escasa, si se tiene en cuenta la cantidad e importancia de estas áreas protegidas y los diversos ecosistemas que abarcan en el territorio nacional, lo que además hace evidente la necesidad de empezar iniciativas de documentación de la biodiversidad, mediante expediciones que permitan recolectar especímenes de las especies presentes allí. Específicamente, hay poca evidencia de recopilación de información histórica de aves en estas áreas protegidas (Álvarez et al, 2003); sin embargo, recientemente Linares-Romero et al. (2020), aportaron un trabajo recopilatorio del PNN Chingaza, donde reportan 531 especies de aves, en un área de 76000 ha. Estos resultados son interesantes, si contrastamos el número de especies halladas en nuestro trabajo (357 spp.), en un área considerada una de las más pequeñas de todos los parques nacionales del país (10 019 ha).

A pesar de los vacíos, existen algunos aportes recientes de varios parques nacionales, que incluyen la Serranía de la Macarena, donde se reportan 145 especies (Losada-Prado et al., 2017); El Cocuy, con 45 especies (Suárez-Sanabria \& Cadena, 2014); Farallones de Cali, con 157 especies (Bermúdez-Vera et al., 2013) y Tatamá, 
Munchique (López-Ordoñez et al., 2013; RamírezBurbano, 2013) y Los Nevados (Acevedo-Charry et al., 2013) con aportes de ecología y nuevos registros de migratorias. De acuerdo a nuestro estudio y al trabajo más reciente de la avifauna de Chiribiquete (Naranjo \& Stiles, 2017), las expediciones son fundamentales para continuar ampliando el conocimiento de la avifauna en aquellos territorios recónditos y aún conservados del país.

En algunos casos, los vacíos de información son debidos a que históricamente los parques nacionales naturales se convirtieron en resguardos para grupos armados, corredores estratégicos o en zonas de disputa en medio del conflicto (Assmus- Correa, 2015; Muñoz- Sáenz, 2015). Samaná y El PNN Selva de Florencia no fueron la excepción a estos hechos; incluso el Sendero S6 del parque (Boquerón-Yarumal), donde obtuvimos registros nuevos para Caldas, que incluyen posibles ampliaciones de distribución (M. lanceolata. P. aureopectus y G. flavirostris) fue recientemente desminado. Su historia de conflicto armado, como la de muchas áreas protegidas del país, dejó zonas inaccesibles, que por muchos años impidieron aportar al conocimiento de su biodiversidad en gran parte de su territorio. A pesar de esto, los primeros estudios de aves realizados en el parque (Corpocaldas \& Universidad de Caldas, 2001; Castellanos et al., 2003) permitieron categorizarlo como AICA (Franco et al., 2009), que evidenció su importancia en términos de conservación de aves.

En general, se ha considerado que la avifauna de la región andina es la más conocida (Estela \& LópezVictoria, 2005; Bravo \& Naranjo, 2006); sin embargo, este estudio demuestra que existen zonas de este territorio que aún no se han explorado lo suficiente y que pueden contribuir al conocimiento de las afinidades biogeográficas de las aves del país. De acuerdo a la revisión de especímenes y con base en el trabajo de Cuervo et al. (2008b), parte de los vacíos de información para algunas especies reportadas aquí (M. lanceolata y G. flavirostris), están relacionados con su escasa representatividad en colecciones biológicas. Por esta razón, es necesario incentivar y aportar información a las colecciones ornitológicas, con el fin de fortalecer el conocimiento de las especies, para esclarecer sus incertidumbres taxonómicas (Cuervo et al., 2006). Particularmente para nuestro estudio es interesante el registro de Pseudotriccus pelzelni (IAvH-A 06445), que inicialmente fue identificado como Ochthoeca diadema, y fue solo identificado correctamente 8 años después de ser recolectado, lo que además evidencia la importancia de recolectar evidencia física de las especies (especímenes de estudio) que puede ser replicada y confirmada en el tiempo.

\section{Agradecimientos}

Agradecemos muy especialmente al director del área protegida PNN Selva de Florencia Hugo Fernando Ballesteros y todo su equipo de trabajo en el parque, Caterine Rodríguez, Alejandro López Maya, Nathalya Henao Muñoz, Amilvia Acosta Castañeda y Santiago Cardona Toro. Al equipo de apoyo de la expedición Aves de Caldas: Paola Sepúlveda, Gustavo Pineda, Víctor Méndez, John Murillo, Laura Alejandra Valencia, Enrick Meza y a Daniel Ocampo y Juan Ruiz, equipo de Vencejo Films. A Beatriz Edilma Toro Restrepo, coordinadora de Caldas Expedición Siglo XXI, los estudiantes del programa de Biología pertenecientes al Semillero de Investigación en Ornitología de la Universidad de Caldas: Alejandra María Patiño Gallego, Nicolás Botero Henao, Juan Felipe León León, Deivy Gómez y al biólogo Daniel Felipe Aristizábal. A Héctor Ramírez Chávez, por el apoyo en la búsqueda de información en la colección ornitológica del Museo de Historia Natural-Centro de Museos de la Universidad de Caldas. Finalmente, a la Universidad de Caldas y Vicerrectorías de Proyección Universitaria y de Investigaciones y Posgrados, a Cotelco, Corpocaldas, CHEC, Aguas de Manizales, Alcaldía de Manizales, La Gobernación de Caldas y la Sociedad Caldense de Ornitología, por el apoyo y financiación en nuestras labores de exploración.

\section{Referencias}

Acevedo-Charry, O., Matta-Camacho, N. E. \& Moncada-Álvarez, L. I. (2013). Registros nuevos o poco conocidos de aves migratorias en la Laguna del Otún, Parque Nacional Natural Los Nevados, Risaralda, Colombia. Acta Biológica Colombiana,18, 191-198.

Álvarez, M., Umaña, A. M., Mejía, G. D., Cajiao, J., Von Hildebrand, P. \& Gast, F. (2003). Aves del Parque Nacional Natural Serranía de Chiribiquete, Amazonia-Provincia de la Guyana, Colombia. Biota Colombiana, 4(1), 49-63.

Assmus-Correa, G. (2015). Restauración ambiental y posconflicto. Revista de la Universidad de La Salle, 66, 133-144. 
Avendaño, J.E., Bohórquez, C.I., Arzuza-Buelvas, L., Estela, F., Cuervo, A.M., Stiles, F.G. \& Renjifo, L.M. (2017). Lista de chequeo de las aves de Colombia: Una síntesis del estado de conocimiento desde Hilty \& Brown (1986). Ornitología Colombiana, 16.

Ayerbe-Quiñones, F. (2018). Guía ilustrada de la Avifauna colombiana. Wildlife Conservation Society (WCS). 212 pp.

Ballesteros, H. F., Herrera- Caicedo, A., EcheverryGarzón, J., Rodríguez-Hurtado, K., HincapiéGiraldo, W., Patiño-Cortes, E., Cardona- Toro, A. F., Lancheros, R. \& Areiza, A. (2017). (Informe técnico). Plan de manejo 2018-2023 Parque Nacional Natural Selva de Florencia. Manizales.: Parques Nacionales Naturales de Colombia, Dirección Territorial Noroccidente. $128 \mathrm{pp}$.

Bermúdez-Vera, J. C., López, S. D., Martínez, M. A. S. \& Tenorio, E. (2013). Avifauna en un área perturbada del bosque andino en el Parque Nacional Natural Farallones de Cali, corregimiento de Pance, Valle del Cauca (Colombia). Biota Colombiana, 14 (3), 3543.

Bravo, G. \& Naranjo, L. G. (2006). Estado del conocimiento sobre las aves terrestres en Colombia. En Chaves, M. E. \& Santamaría, M (Eds.). Informe sobre el avance en el conocimiento y la información de la biodiversidad 1998-2004. Tomo II. Pp: 130-151. Bogotá D.C.: Instituto de Investigación de Recursos Biológicos Alexander von Humboldt.

Castaño-Hernández, J. \& Calderón-Franco, D. (2012). First record of Scaled Fruiteater Ampelioides tschudii in the Central Andes of Colombia. Cotinga. 34,59-160.

Castaño-Villa, G. J. \& Patiño-Zabala, J. C. (2008). Extinciones locales de aves en fragmentos de bosque en la región de Santa Elena, Andes Centrales, Colombia. Hornero, 23(1), 23-34.

Castellanos, O., Aristizábal, A. \& Betancourt, A.F. (2003). Inventario de la avifauna perteneciente a la Selva de Florencia (cuenca del río San Antonio y del río Hondo) Samaná, Caldas. Boletín Científico Museo Historia Natural Universidad de Caldas, 7, 15-26.

Chaparro-Herrera, S., Córdoba-Córdoba, S., LópezOrdóñez, J. P., Restrepo-Cardona, J. S. \& CortésHerrera, O. (2015). Los búhos de Colombia. En Enríquez, P. L. (Ed.). Los Búhos Neotropicales. Diversidad y Conservación. Pp: 277-329. Chiapas.: Ecosur.

Corpocaldas \& Asociación Calidris. (2010). Estado de conocimiento de las aves en el departamento de Caldas: Prioridades de conservación y vacios de información. (Informe técnico). Manizales.: Corporación Autónoma Regional de Caldas. 105 pp.
Corpocaldas \& Universidad de Caldas. (2001). Informe final sobre biodiversidad de la Selva de Florencia. (Informe técnico). Manizales.: Universidad de Caldas.

Cuadros, T. (1988). Aspectos ecológicos de la comunidad de aves en un bosque nativo en la Cordillera Central de Antioquia (Colombia). Hornero, 13(01), 008-020.

Cuervo, A. M., Pulgarín, P. C., Calderón-F, D., Ochoa-Quintero, J. M., Delgado-V, C. A., Palacio, A., Botero, J. M. \& Múnera, W. A. (2008a). Avifauna of the northern Cordillera Central of the Andes, Colombia. Ornitologia Neotropical, 19(4), 495-515.

Cuervo, A. M., Pulgarín, P. C. \& Calderón, D. (2008b). New distributional bird data from the Cordillera Central of the Colombian Andes, with implications for the biogeography of northwestern South America. The Condor, 110(3), 526-537. https:// doi.org/10.1525/cond.2008.8555

Cuervo, A. M., Cadena, C. D. \& Parra, J. L. (2006). Seguir colectando aves en Colombia es imprescindible: un llamado a fortalecer las colecciones ornitológicas. Ornitología Colombiana, 4, 51-58.

Delgado-V., C. A. (2002). Observations of the Ochre-breasted (Grallaricula flavirostris) and Slate-crowned (G. nana) Antpittas in Colombia. Ornitología Neotropical, 13, 423-425.

Donegan, T. M. (2012). Range extensions and other notes on the birds and conservation of the Serranía de San Lucas, an isolated mountain range in northern Colombia. Bulletin of the British Ornithologists' Club, 132 (3), 140-161.

Estela, F. A. \& López-Victoria, M. (2005). Aves de la parte baja del río Sinú, Caribe colombiano: inventario y ampliaciones de distribución. Boletín de Investigaciones Marinas y Costeras, 34(1), 7-42. https:// doi.org/10.25268/bimc.invemar.2005.34.0.232 Franco, A. M., Devenish, C., Barrero, M. C. \& Romero, M. H. (2009). En Devenish, C., Díaz Fernández, D. F., Clay, R. P., Davidson, I. \& Yépez- Zabala. I (Eds.). Important Bird Areas Americas - Priority sites for biodiversity conservation. Pp 135 -148. Quito, Ecuador: BirdLife International (BirdLife Conservation Series No. 16).

Franco, A. M. \& Bravo, G. (2005). Áreas importantes para la conservación de las aves en Colombia. Áreas importantes para la conservación de las aves en los Andes tropicales: sitios prioritarios para la conservación de la biodiversidad. Quito: BirdLife International \& Conservation International. 117-281 pp.

Herrera Caicedo A., Echeverry-Garzón, J., Roncancio, N., Patiño Cortés, E., Hincapié-Giraldo, W., Cardona-Toro, A. \& Lancheros Murillo, R. (2016). Portafolio 
de proyectos de Investigación Parque Nacional Natural Selva de Florencia. Plan de Manejo 2016-2021 del PNN Selva de Florencia. (Informe técnico). Manizales.: Parques Nacionales Naturales de Colombia. Dirección Territorial Andes Occidentales. 21 pp.

Hilty, S. L. \& Brown, W.L. (2001). Guía de las Aves de Colombia. Traducción Álvarez-López, H. Cali.: American Bird Conservancy. 467 pp.

IDEAM., IGAC., IAvH., Invemar., I. Sinchi. \& IIAP. (2007). Ecosistemas continentales, costeros y marinos de Colombia. Bogotá D.C.: Instituto de Hidrología, Meteorología y Estudios Ambientales (Ideam), Instituto Geográfico Agustín Codazzi (IGAC), Instituto de Investigación de Recursos Biológicos Alexander von Humboldt (IAvH), Instituto de Investigaciones Ambientales del Pacífico John von Neumann, (IIAP), Instituto de Investigaciones Marinas y Costeras José Benito Vives de Andréis (Invemar) e Instituto Amazónico de Investigaciones Científicas (SINCHI). 276 pp.

Kattan, G., Franco, P., Saavedra-Rodríguez, C. A., Valderrama, C., Rojas, V., Osorio, D. \& Martínez, J. (2006). Spatial components of bird diversity in the Andes of Colombia: Implications for designing a regional reserve system. Conservation Biology, 20, 1203-1211. https://doi.org/10.1111/j.1523-1739.2006.00402.x

Linares-Romero, L. G., Acevedo-Charry, O., Avellaneda, A., Cortés-Herrera, O., Cuervo, A., Galindo-T, R., Hernández, D., Pérez-Peña, S., Ramiro-Pulido, A., Pulido-Santacruz, P., Santana, D., Seeholzer, G., Sierra-Buitrago, M. S., Soto-Patiño, J. \& Laverde-R, O. (2020). Aves del Parque Nacional Natural Chingaza y zona de amortiguación, Cordillera Oriental de Colombia. Biota Colombiana, 21(1), 117-129. https:// doi.org/10.21068/c2020.v21n01a09

Londoño-Betancourth, J. C. (2011). Una mirada a la diversidad ornitológica de Pereira. Boletín Científico Museo de Historia Natural, 15(1), 84-103.

López-Ordóñez, J. P., Cortés-Herrera, J. O., Páez-Ortíz, C.A. \& González-Rojas, M. F (2013). Nuevos registros y comentarios sobre la distribución de algunas especies de aves en los Andes Occidentales de Colombia. Ornitología Colombiana, 13.

Losada-Prado, S., Tenorio, E. A. \& Alfonso Bernal, M. F. (2017). Aves de La Serranía de la Macarena. En Lasso, C. A. \& Morales-Betancourt, M. A (Eds.). Fauna de Caño Cristales, Sierra La Macarena, Meta, Colombia. Pp 124-135. Bogotá D.C.: Serie Editorial Fauna Silvestre Neotropical. Instituto de Investigación de Recursos Biológicos Alexander von Humboldt (IAvH).
Lozada, S. \& Molina, Y. (2011). Avifauna del bosque seco tropical en el departamento del Tolima (Colombia): Análisis de la comunidad. Caldasia, 33(1).

Márquez, C., Bechard, M., Gast, F. \& Vanegas, V.H. (2005). Aves rapaces diurnas de Colombia. Bogotá D.C.: Instituto de Investigación de Recursos Biológicos Alexander von Humboldt. 394 pp.

Mcmullan, M., Donegan, T. M. \& Quevedo, A. (2011). Guía de Campo de las Aves de Colombia. Fundación ProAves. $250 \mathrm{pp}$.

Muñoz-Sáenz, M. de C. (2015). Desafios de la gestión ambiental frente a la transición hacia la paz en Colombia: propuesta de gestión. (Trabajo de grado). Bogotá D. C.: Pontificia Universidad Javeriana, Facultad de Estudios Ambientales y Rurales. 165 pp.

Naranjo, L. G. \& Stiles, G. (2017). La avifauna del Parque Nacional Natural Chiribiquete: resultados de tres expediciones recientes a sectores previamente inexplorados. Revista Amazónica Colombiana, 10, 141-160.

Naranjo, L.G., Amaya, J. D., Eusse-González, D. \& Cifuentes-Sarmiento. Y. (2012). Guía de las Especies Migratorias de la Biodiversidad en Colombia-Aves. Volumen 1. Bogotá D.C.: WWF Colombia. Ministerio de Ambiente y Desarrollo Sostenible. 708 pp.

Olaciregui, C. \& Urueña, L. E. (2011). First nesting record of Blue-fronted Parrotlet Touit dilectissimus, with some ecological notes. Cotinga, 33, 84-86.

Paiba-Alzate, J. E., López-Orozco, N. \& Betancourt, A. F. (2010). New bird records at Parque Nacional Natural Selva de Florencia, Caldas. Boletín Científico. Centro de Museos. Museo de Historia Natural, 14(1), 114-120.

Peña, R. M. (1998). La hormiguera Grallaricula flavirostris en el municipio de San Carlos. Cucarachero, 38, 9.

Ralph, C. J., Geoffrey, R. G., Pyle, P., Martin, T. E., DeSante, D. F. \& Milá, B. (1996). Manual de métodos de campo para el monitoreo de aves terrestres. Albany, CA.: US Department of Agriculture, Forest Service, Pacific Southwest Research Station. 51 pp.

Ramírez Burbano, M.B. (2013). Redes de interacción mutualista colibrí-flor en el Parque Nacional Natural Munchique: ¿La pérdida de un colibrí endémico y en peligro crítico de extinción, acarrea el colapso del sistema? (Trabajo de grado). Bogotá D. C.: Universidad Nacional de Colombia, Facultad de Ciencias, Departamento de Biología. 102 pp.

Remsen, J. V., Areta, J. I., Bonaccorso, E., Claramunt, S., Jaramillo, A., Pacheco, J. F., Robbins, M. B., Stiles, F. G., Stotz, D. F. \& Zimmer, K. J. Version 
(abril 2020). A classification of the bird species of South America. American Ornithological Society. https:// bit.ly/2PAPYFG

Renjifo, L. M., Gómez, M. F., Velasquez-Tibata, J., Amaya-Villarreal, A. M., Kattan, G. H., Amaya-Espinel, J. D. \& Burbano- Girón, J. (2014). Libro rojo de aves de Colombia, Volumen I: bosques húmedos de los Andes y la costa Pacífica. Bogotá D.C.: Editorial Pontificia Universidad Javeriana e Instituto Alexander von Humboldt. 466 pp.

Restall, R., Rodner, C. \& Lentino, M. (2007). Birds of Northern South America- Identification Guide. New heaven, USA.: Yale University Press. 880 pp.

Rodríguez, N., Armenteras, D., Morales, M. \& Romero, M. (2006). Ecosistemas de los Andes colombianos. Segunda edición. Bogotá D.C.: Instituto de Investigación de Recursos Biológicos Alexander von Humboldt. $154 \mathrm{pp}$.

Rodríguez-Mahecha, J. V. \& Hernández- Camacho, J. (2002). Loros de Colombia. Bogotá D.C.: Conservación Internacional.

Stiles, G. \& Bohórquez, C. I. (2000). Evaluando el estado de la biodiversidad: el caso de la avifauna de la Serranía de las Quinchas, Boyacá, Colombia. Caldasia, 22(1), 61-92.

Suárez-Sanabria, N. \& Cadena, C. D. (2014). Diversidad y estructura de la avifauna del Valle de Lagunillas, Parque Nacional Natural El Cocuy, Colombia. Ornitología Colombiana, 14.

Terborgh, J. (1971). Distribution on environmental gradients: theory and a preliminary interpretation of distributional patterns in the avifauna of the Cordillera Vilcabamba, Perú. Ecology, 52, 23-40.

Villarreal, H., Álvarez, M., Córdoba, S., Escobar, F., Fagua, G., Gast, F., Mendoza, H., Ospina, M. \& Umaña, A.M (2006). Manual de métodos para el desarrollo de inventarios de biodiversidad. Programa de Inventarios de Biodiversidad. Segunda edición. Bogotá D.C.:
Instituto de Investigación de Recursos Biológicos Alexander von Humboldt. 236 pp.

\section{Lista de Figuras}

Figura 1. PNN Selva de Florencia ubicado en la vertiente oriental de la Cordillera Central, en el departamento de Caldas entre los municipios Pensilvania y Samaná. Puntos de muestreo, azul: Sector Pensilvania; rojo: Sector Florencia; violeta: Sector Samaná, amarillo: Expedición actualización aves de Caldas (2018); rosado: Caldas Expedición Siglo XXI.

Figura 2. Cobertura vegetal asociada a los distritos de manejo del PNN Selva de Florencia. A y B- Sector Samaná. C y D- Sector Florencia. E y F- Sector Pensilvania. Fotografías: Felipe Cardona.

Figura 3. Fotografía de cámara trampa de la perdiz collareja (Odontophorus erythrops). Fotografías: Archivo Parques Nacionales Naturales.

Figura 4. Fotografía del búho crestado (Lophostrix cristata) en la Vereda El Quindío (Sector Samaná). Fotografía: Milton Pineda.

Figura 5. Algunas aves del del Parque Nacional Natural Selva de Florencia, Caldas, Colombia: A, bigotudo lanceolado (Micromonacha lanceolata); B, tororoi piquigualdo (Grallaricula flavirostris); C, frutero pechidorado (Pipreola aureopectus); D, frutero escamado (Ampelioides tschudii) E, musguerito gargantilla (Iridosornis porphyrocephalus); F, picogordo pizarra (Saltator grossus); G, pinzón oliva (Arremon castaneiceps);. Fotografías: Archivo Parques Nacionales Naturales. A y E, Milton Pineda Duque; B, C y D, Felipe Cardona Toro; F, Alejandra María Patiño Gallego; G, Santiago Cardona Toro

Figura 6. Registro de S. ornatus y su nido en el PNN Selva de Florencia. Fotografías: Nicolás Botero Henao. 


\section{Lista de Anexos}

Anexo 1. Especies de aves registradas en el Parque Nacional Natural Selva de Florencia, Samaná (Caldas). (*) Nuevos registros para el Parque y el departamento. Nombre común (Mcmullan et al., 2011). Categoría de amenaza global (IUCN) y nacional (Libro rojo): NT (Casi Amenazado), VU (Vulnerable), EN (En Peligro). Información de la especie: EN (Endémica), MB (Migratoria Boreal), MBr (Migratoria Boreal con poblaciones reproductivas), ML (Migrante local) según Naranjo et al. (2012).

\begin{tabular}{|c|c|c|c|c|c|c|}
\hline Orden & Familia / Especie & Nombre de autor & Nombre común & $\begin{array}{c}\text { Amenaza } \\
\text { global } \\
\text { (IUCN) }\end{array}$ & $\begin{array}{c}\text { Amenaza } \\
\text { nacional } \\
\text { (Libro } \\
\text { rojo) }\end{array}$ & $\begin{array}{c}\text { Info. } \\
\text { de la } \\
\text { especie }\end{array}$ \\
\hline \multirow[t]{2}{*}{ Tinamiformes } & Tinamidae & & & & & \\
\hline & Crypturellus soui & (Hermann, 1783) & Tinamú chico & & & \\
\hline \multirow[t]{2}{*}{ Anatidae } & Anatidae & & & & & \\
\hline & Merganetta armata & Gould, 1842 & Pato de torrente & & & \\
\hline \multirow[t]{7}{*}{ Galliformes } & Cracidae & & & & & \\
\hline & Chamaepetes goudotii & (Lesson, 1828) & Pava maraquera & & & \\
\hline & Aburria aburri & (Lesson, 1828) & Pava negra & NT & & \\
\hline & Ortalis columbiana & Hellmayr, 1906 & $\begin{array}{l}\text { Guacharaca } \\
\text { colombiana }\end{array}$ & & & $\mathrm{EN}$ \\
\hline & Odontophoridae & & & & & \\
\hline & $\begin{array}{l}\text { Odontophorus } \\
\text { erythrops* }\end{array}$ & Gould, 1859 & Perdiz collareja & & & \\
\hline & $\begin{array}{l}\text { Odontophorus } \\
\text { hyperythrus }\end{array}$ & Gould, 1858 & Perdiz colorada & NT & & $\mathrm{EN}$ \\
\hline \multirow[t]{10}{*}{ Columbiformes } & Columbidae & & & & & \\
\hline & Patagioenas speciosa* & (Gmelin, 1789) & Paloma escamada & & & \\
\hline & Patagioenas fasciata & (Say, 1823) & Paloma collareja & & & ML \\
\hline & $\begin{array}{l}\text { Patagioenas } \\
\text { cayennensis }\end{array}$ & (Bonnaterre, 1792) & Paloma morada & & & \\
\hline & $\begin{array}{l}\text { Patagioenas } \\
\text { subvinacea }\end{array}$ & (Lawrence, 1868) & Paloma colorada & VU & & ML \\
\hline & Geotrygon montana & (Linnaeus, 1758) & $\begin{array}{l}\text { Paloma perdiz } \\
\text { rojiza }\end{array}$ & & & \\
\hline & Leptotila verreauxi & (Bonaparte, 1855) & Tórtola colipinta & & & \\
\hline & Zentrygon frenata & (Tschudi, 1843) & $\begin{array}{l}\text { Paloma perdiz } \\
\text { bigotuda }\end{array}$ & & & \\
\hline & Zentrygon linearis & (Prévost, 1843) & $\begin{array}{l}\text { Paloma perdiz } \\
\text { lineada }\end{array}$ & & & \\
\hline & Zenaida auriculata & (Des Murs, 1847) & $\begin{array}{l}\text { Torcaza } \\
\text { naguiblanca }\end{array}$ & & & \\
\hline
\end{tabular}




\begin{tabular}{|c|c|c|c|c|c|c|}
\hline Orden & Familia / Especie & Nombre de autor & Nombre común & $\begin{array}{c}\text { Amenaza } \\
\text { global } \\
\text { (IUCN) }\end{array}$ & $\begin{array}{c}\text { Amenaza } \\
\text { nacional } \\
\text { (Libro } \\
\text { rojo) }\end{array}$ & $\begin{array}{c}\text { Info. } \\
\text { de la } \\
\text { especie }\end{array}$ \\
\hline & Claravis pretiosa & $\begin{array}{l}\text { (Ferrari-Pérez, } \\
\text { 1886) }\end{array}$ & Tortolita azul & & & \\
\hline \multirow[t]{4}{*}{ Cuculiformes } & Cuculidae & & & & & \\
\hline & Crotophaga ani & Linnaeus, 1758 & $\begin{array}{l}\text { Garrapatero } \\
\text { piquiliso }\end{array}$ & & & \\
\hline & Tapera naevia & (Linnaeus, 1766) & Cuco sin fín & & & \\
\hline & Piaya cayana & (Linnaeus, 1766) & $\begin{array}{l}\text { Cuco ardilla } \\
\text { común }\end{array}$ & & & \\
\hline \multirow[t]{2}{*}{$\begin{array}{l}\text { Steatornithifor- } \\
\text { mes }\end{array}$} & Steatornithidae & & & & & \\
\hline & Steatornis caripensis & Humboldt, 1817 & Guácharo común & & & \\
\hline \multirow[t]{2}{*}{ Nyctibiiformes } & Nyctibiidae & & & & & \\
\hline & Nyctibius griseus & (Gmelin, 1789) & Bien parado común & & & \\
\hline \multirow[t]{2}{*}{$\begin{array}{l}\text { Caprimulgifor- } \\
\text { mes }\end{array}$} & Caprimulgidae & & & & & \\
\hline & $\begin{array}{l}\text { Nyctidromus } \\
\text { albicollis }\end{array}$ & (Gmelin, 1789) & Bujío & & & \\
\hline \multirow[t]{13}{*}{ Apodiformes } & Apodidae & & & & & \\
\hline & Streptoprocne zonaris & (Shaw, 1796) & Vencejo collarejo & & & \\
\hline & Streptoprocne rutila & (Vieillot, 1817) & vencejo cuellirrojo & & & \\
\hline & Chaetura brachyura & (Jardine, 1846) & Vencejo rabicorto & & & \\
\hline & Trochilidae & & & & & \\
\hline & Florisuga mellivora & (Linnaeus, 1758) & $\begin{array}{l}\text { Colibrí } \\
\text { nuquiblanco }\end{array}$ & & & \\
\hline & Eutoxeres aquila & (Bourcier, 1847) & $\begin{array}{l}\text { Pico de hoz- } \\
\text { coliverde }\end{array}$ & & & ML \\
\hline & Threnetes ruckeri & (Bourcier, 1847) & $\begin{array}{l}\text { Ermitaño } \\
\text { coliblanco }\end{array}$ & & & \\
\hline & $\begin{array}{l}\text { Phaethornis } \\
\text { striigularis }\end{array}$ & Gould, 1854 & $\begin{array}{l}\text { Ermitaño } \\
\text { gorgirrayado }\end{array}$ & & & \\
\hline & $\begin{array}{l}\text { Phaethornis } \\
\text { anthophilus }\end{array}$ & (Bourcier, 1843) & Ermitaño carinegro & & & \\
\hline & Phaethornis guy & (Lesson, 1833) & Ermitaño verde & & & ML \\
\hline & $\begin{array}{l}\text { Phaethornis syrmato- } \\
\text { phorus }\end{array}$ & Gould, 1851 & Ermitaño aleonado & & & \\
\hline & Doryfera ludovicae & $\begin{array}{l}\text { (Bourcier \& } \\
\text { Mulsant, 1847) }\end{array}$ & $\begin{array}{l}\text { Pico de lanza frenti } \\
\text { verde }\end{array}$ & & & \\
\hline
\end{tabular}




\begin{tabular}{|c|c|c|c|c|c|c|}
\hline Orden & Familia / Especie & Nombre de autor & Nombre común & $\begin{array}{l}\text { Amenaza } \\
\text { global } \\
\text { (IUCN) }\end{array}$ & $\begin{array}{c}\text { Amenaza } \\
\text { nacional } \\
\text { (Libro } \\
\text { rojo) }\end{array}$ & $\begin{array}{l}\text { Info. } \\
\text { de la } \\
\text { especie }\end{array}$ \\
\hline & Schistes geoffroyi & (Bourcier, 1843) & Colibrí piquicuña & & & \\
\hline & Colibri delphinae & (Lesson, 1839) & Colibrí pardo & & & ML \\
\hline & Colibri cyanotus & (Swainson, 1827) & Colibrí verde & & & \\
\hline & Colibri coruscans & (Gould, 1846) & Colibrí chillón & & & \\
\hline & Heliothryx barroti & (Bourcier, 1843) & Hada coliblanca & & & \\
\hline & Discosura conversii ${ }^{*}$ & $\begin{array}{l}\text { (Bourcier \& } \\
\text { Mulsant, 1846) }\end{array}$ & Cola de lira verde & & & \\
\hline & $\begin{array}{l}\text { Adelomyia } \\
\text { melanogenys }\end{array}$ & (Fraser, 1840) & $\begin{array}{l}\text { Colibrí } \\
\text { pechipunteado }\end{array}$ & & & \\
\hline & Aglaiocercus kingii & (Lesson, 1832) & Cometa verdiazul & & & \\
\hline & $\begin{array}{l}\text { Haplophaedia } \\
\text { aureliae }\end{array}$ & $\begin{array}{l}\text { (Bourcier \& } \\
\text { Mulsant, 1846) }\end{array}$ & $\begin{array}{l}\text { Calzoncitos } \\
\text { verdoso }\end{array}$ & & & \\
\hline & Coeligena Coeligena & (Lesson, 1833) & Inca bronceado & & & \\
\hline & $\begin{array}{l}\text { Boissonneaua } \\
\text { flavescens }\end{array}$ & (Loddiges, 1832) & Colibrí chupasavia & & & \\
\hline & $\begin{array}{l}\text { Ocreatus } \\
\text { underwoodii }\end{array}$ & (Lesson, 1832) & $\begin{array}{l}\text { Cola de raqueta } \\
\text { pierniblanco }\end{array}$ & & & \\
\hline & Heliodoxa jacula & Gould, 1850 & $\begin{array}{l}\text { Diamante frenti- } \\
\text { verde }\end{array}$ & & & \\
\hline & $\begin{array}{l}\text { Chaetocercus } \\
\text { mulsant }\end{array}$ & Bourcier, 1842 & $\begin{array}{l}\text { Rumbito } \\
\text { buchiblanco }\end{array}$ & & & \\
\hline & Chlorostilbon gibsoni & (Fraser, 1840) & $\begin{array}{l}\text { Esmeralda } \\
\text { piquirroja }\end{array}$ & & & \\
\hline & Chalybura buffonii & (Lesson, 1832) & Colibrí de buffon & & & \\
\hline & Thalurania colombica & (Bourcier, 1843) & Ninfa coroniazul & & & \\
\hline & $\begin{array}{l}\text { Saucerottia } \\
\text { saucerottei }\end{array}$ & $\begin{array}{l}\text { (DeLattre and } \\
\text { Bourcier, 1846) }\end{array}$ & Amazilia coliazul & & & \\
\hline & Amazilia tzacatl & (De la Llave, 1833) & Amazilia colirrufa & & & \\
\hline & Uranomitra franciae & $\begin{array}{l}\text { (Bourcier and } \\
\text { Mulsant, 1846) }\end{array}$ & Amazilia andina & & & \\
\hline & Chlorestes julie & (Bourcier, 1843) & Colibrí pechiverde & & & \\
\hline \multirow[t]{2}{*}{$\begin{array}{l}\text { Charadriifor- } \\
\text { mes }\end{array}$} & Charadriidae & & & & & \\
\hline & Vanellus chilensis & (Molina, 1782) & Alcaraván & & & \\
\hline \multirow[t]{2}{*}{ Cathartiformes } & Cathartidae & & & & & \\
\hline & Sarcoramphus papa & (Linnaeus, 1758) & $\begin{array}{l}\text { Rey de los } \\
\text { gallinazos }\end{array}$ & & & \\
\hline
\end{tabular}




\begin{tabular}{|c|c|c|c|c|c|c|}
\hline Orden & Familia / Especie & Nombre de autor & Nombre común & $\begin{array}{c}\text { Amenaza } \\
\text { global } \\
\text { (IUCN) }\end{array}$ & $\begin{array}{c}\text { Amenaza } \\
\text { nacional } \\
\text { (Libro } \\
\text { rojo) }\end{array}$ & $\begin{array}{c}\text { Info. } \\
\text { de la } \\
\text { especie }\end{array}$ \\
\hline & Coragyps atratus & (Bechstein, 1793) & Gallinazo común & & & \\
\hline & Cathartes aura & (Linnaeus, 1758) & Guala común & & & \\
\hline \multirow[t]{12}{*}{ Accipitriformes } & Pandionidae & & & & & \\
\hline & Pandion haliaetus & (Linnaeus, 1758) & Águila pescadora & & & $\mathrm{MBr}$ \\
\hline & Accipitridae & & & & & \\
\hline & Elanoides forficatus & (Linnaeus, 1758) & Aguililla tijereta & & & $\mathrm{MBr}$ \\
\hline & Spizaetus tyrannus & $\begin{array}{l}\text { (Wied-Neuwied, } \\
\text { 1820) }\end{array}$ & Águila iguanera & & & \\
\hline & Spizaetus ornatus & (Daudin, 1800) & Águila coronada & NT & & \\
\hline & Harpagus bidentatus & (Latham, 1790) & Gavilán lagartero & & & \\
\hline & Accipiter striatus & Vieillot, 1808 & Azor cordillerano & & & \\
\hline & $\begin{array}{l}\text { Morphnarchus } \\
\text { princeps }\end{array}$ & (P. L. Sclater, 1865) & Gavilán príncipe & & & \\
\hline & $\begin{array}{l}\text { Rupornis } \\
\text { magnirostris }\end{array}$ & (Gmelin, 1788) & Gavilán caminero & & & \\
\hline & $\begin{array}{l}\text { Geranoaetus } \\
\text { albicaudatus }\end{array}$ & Vieillot, 1816 & Gavilán coliblanco & & & \\
\hline & Buteo platypterus & (Vieillot, 1823) & Gavilán aliancho & & & $\mathrm{MB}$ \\
\hline \multirow[t]{7}{*}{ Strigiformes } & Tytonidae & & & & & \\
\hline & Tyto alba & (Scopoli, 1769) & Lechuza & & & \\
\hline & Strigidae & & & & & \\
\hline & Megascops choliba & (Vieillot, 1817) & Currucutú & & & \\
\hline & Lophostrix cristata* & (Daudin, 1800) & Búho crestado & & & \\
\hline & $\begin{array}{l}\text { Pulsatrix } \\
\text { perspicillata }\end{array}$ & (Latham, 1790) & Búho de anteojos & & & \\
\hline & Ciccaba virgata & (Cassin, 1849) & Búho moteado & & & \\
\hline \multirow[t]{4}{*}{ Trogoniformes } & Trogonidae & & & & & \\
\hline & $\begin{array}{l}\text { Pharomachrus } \\
\text { auriceps }\end{array}$ & (Gould, 1842) & Quetzal colinegro & & & \\
\hline & Trogon collaris & Vieillot, 1817 & Trogón collarejo & & & ML \\
\hline & Trogon personatus & Gould, 1842 & $\begin{array}{l}\text { Trogón } \\
\text { enmascarado }\end{array}$ & & & \\
\hline \multirow[t]{2}{*}{ Coraciiformes } & Momotidae & & & & & \\
\hline & $\begin{array}{l}\text { Momotus aequato- } \\
\text { rialis }\end{array}$ & Gould, 1858 & $\begin{array}{l}\text { Barranquero } \\
\text { andino }\end{array}$ & & & \\
\hline
\end{tabular}




\begin{tabular}{|c|c|c|c|c|c|c|}
\hline Orden & Familia / Especie & Nombre de autor & Nombre común & $\begin{array}{l}\text { Amenaza } \\
\text { global } \\
\text { (IUCN) }\end{array}$ & $\begin{array}{c}\text { Amenaza } \\
\text { nacional } \\
\text { (Libro } \\
\text { rojo) }\end{array}$ & $\begin{array}{l}\text { Info. } \\
\text { de la } \\
\text { especie }\end{array}$ \\
\hline \multirow{8}{*}{ Galbuliformes } & \multicolumn{6}{|l|}{ Alcedinidae } \\
\hline & $\begin{array}{l}\text { Chloroceryle } \\
\text { americana }\end{array}$ & (Gmelin, 1788) & $\begin{array}{l}\text { Martin pescador } \\
\text { chico }\end{array}$ & & & \\
\hline & \multicolumn{6}{|l|}{ Galbulidae } \\
\hline & Galbula ruficauda & Cuvier, 1816 & Jacamar colirufo & & & \\
\hline & \multicolumn{6}{|l|}{ Bucconidae } \\
\hline & Nystalus radiatus & (P. L. Sclater, 1854) & Bobo barrado & & & \\
\hline & $\begin{array}{l}\text { Malacoptila } \\
\text { mystacalis }\end{array}$ & (Lafresnaye, 1850) & Bigotudo canoso & & & \\
\hline & $\begin{array}{l}\text { Micromonacha } \\
\text { lanceolata* }\end{array}$ & (Deville, 1849) & $\begin{array}{l}\text { Bigotudo } \\
\text { lanceolado }\end{array}$ & & & \\
\hline \multirow[t]{18}{*}{ Piciformes } & \multicolumn{6}{|l|}{ Capitonidae } \\
\hline & Capito hypoleucus & Salvin, 1897 & Torito dorsiblanco & VU & & EN \\
\hline & Eubucco bourcierii & (Lafresnaye, 1845) & Torito cabecirrojo & & & ML \\
\hline & \multicolumn{6}{|l|}{ Ramphastidae } \\
\hline & $\begin{array}{l}\text { Ramphastos } \\
\text { vitellinus }\end{array}$ & Lichtenstein, 1823 & Tucán pechiblanco & & & \\
\hline & $\begin{array}{l}\text { Aulacorhynchus } \\
\text { prasinus }\end{array}$ & (Gould, 1833) & $\begin{array}{l}\text { Tucancito } \\
\text { esmeralda }\end{array}$ & & & ML \\
\hline & $\begin{array}{l}\text { Aulacorhynchus } \\
\text { haematopygus }\end{array}$ & (Gould, 1835) & Tucancito rabirrojo & & & \\
\hline & \multicolumn{6}{|l|}{ Picidae } \\
\hline & Picumnus olivaceus & Lafresnaye, 1845 & $\begin{array}{l}\text { Carpinterito } \\
\text { oliváceo }\end{array}$ & & & \\
\hline & $\begin{array}{l}\text { Melanerpes } \\
\text { formicivorus }\end{array}$ & (Swainson, 1827) & $\begin{array}{l}\text { Carpintero de } \\
\text { robledales }\end{array}$ & & & \\
\hline & Melanerpes pulcher & P. L. Sclater, 1870 & Carpintero bonito & & & EN \\
\hline & $\begin{array}{l}\text { Melanerpes } \\
\text { rubricapillus }\end{array}$ & (Cabanis, 1862) & Carpintero habado & & & \\
\hline & Veniliornis kirkii & (Malherbe, 1845) & Carpintero rabirojo & & & \\
\hline & Veniliornis dignus & $\begin{array}{l}\text { (P. L. Sclater \& } \\
\text { Salvin, 1877) }\end{array}$ & $\begin{array}{l}\text { Carpintero } \\
\text { buchiamarillo }\end{array}$ & & & \\
\hline & Campephilus pollens & (Bonaparte, 1845) & Carpintero gigante & & & \\
\hline & $\begin{array}{l}\text { Campephilus } \\
\text { melanoleucos }\end{array}$ & (Gmelin, 1788) & Carpintero marcial & & & \\
\hline & Dryocopus lineatus & (Linnaeus, 1766) & Carpintero real & & & \\
\hline & Colaptes rubiginosus & (Swainson, 1820) & $\begin{array}{l}\text { Carpintero } \\
\text { ahumado }\end{array}$ & & & \\
\hline
\end{tabular}




\begin{tabular}{|c|c|c|c|c|c|c|}
\hline Orden & Familia / Especie & Nombre de autor & Nombre común & $\begin{array}{c}\text { Amenaza } \\
\text { global } \\
\text { (IUCN) }\end{array}$ & $\begin{array}{c}\text { Amenaza } \\
\text { nacional } \\
\text { (Libro } \\
\text { rojo) }\end{array}$ & $\begin{array}{c}\text { Info. } \\
\text { de la } \\
\text { especie }\end{array}$ \\
\hline & Colaptes punctigula & (Boddaert, 1783) & $\begin{array}{l}\text { Carpintero } \\
\text { buchipecoso }\end{array}$ & & & \\
\hline \multirow[t]{7}{*}{ Falconiformes } & Falconidae & & & & & \\
\hline & $\begin{array}{l}\text { Herpetotheres } \\
\text { cachinnans }\end{array}$ & (Linnaeus, 1758) & Halcón culebrero & & & \\
\hline & Caracara cheriway & (Jacquin, 1784) & Caracara moñudo & & & \\
\hline & Milvago chimachima & (Vieillot, 1816) & Pigua & & & \\
\hline & Falco sparverius & Linnaeus, 1758 & $\begin{array}{l}\text { Cernícalo } \\
\text { americano }\end{array}$ & & & \\
\hline & Falco columbarius & Linnaeus, 1758 & Esmerejón & & & MB \\
\hline & Falco rufigularis & Daudin, 1800 & $\begin{array}{l}\text { Halcón } \\
\text { murcielaguero }\end{array}$ & & & \\
\hline \multirow[t]{7}{*}{ Psittaciformes } & Psittacidae & & & & & \\
\hline & Touit dilectissimus* & $\begin{array}{l}\text { (P. L. Sclater and } \\
\text { Salvin, 1871) }\end{array}$ & Cotorrrita cariazul & & & \\
\hline & Pyrilia pyrilia* & (Bonaparte, 1853) & $\begin{array}{l}\text { Cotorra } \\
\text { cariamarilla }\end{array}$ & NT & & \\
\hline & Pionus menstruus & (Linnaeus, 1766) & Cotorra cabeciazul & & & \\
\hline & Pionus chalcopterus & (Fraser, 1841) & Cotorra oscura & & & \\
\hline & $\begin{array}{l}\text { Amazona } \\
\text { mercenarius }\end{array}$ & (Tschudi, 1844) & Lora andina & & & \\
\hline & Forpus conspicillatus & (Lafresnaye, 1848) & $\begin{array}{l}\text { Periquito de } \\
\text { anteojos }\end{array}$ & & & \\
\hline \multirow[t]{8}{*}{ Passeriformes } & Thamnophilidae & & & & & \\
\hline & $\begin{array}{l}\text { Cymbilaimus } \\
\text { lineatus }\end{array}$ & (Leach, 1814) & Batará capirotado & & & \\
\hline & $\begin{array}{l}\text { Thamnophilus } \\
\text { doliatus }\end{array}$ & (Linnaeus, 1764) & Batará barrado & & & \\
\hline & $\begin{array}{l}\text { Thamnophilus } \\
\text { multistriatus }\end{array}$ & Lafresnaye, 1844 & Batará carcajada & & & \\
\hline & $\begin{array}{l}\text { Thamnophilus } \\
\text { atrinucha }\end{array}$ & $\begin{array}{l}\text { Salvin \& Godman, } \\
1892\end{array}$ & Batará occidental & & & \\
\hline & $\begin{array}{l}\text { Thamnophilus } \\
\text { unicolor }\end{array}$ & (P. L. Sclater, 1859) & Batará unicolor & & & \\
\hline & $\begin{array}{l}\text { Dysithamnus } \\
\text { mentalis }\end{array}$ & (Temminck, 1823) & $\begin{array}{l}\text { Hormiguero } \\
\text { tiznado }\end{array}$ & & & ML \\
\hline & $\begin{array}{l}\text { Epinecrophylla } \\
\text { fulviventris }\end{array}$ & (Lawrence, 1862) & $\begin{array}{l}\text { Hormiguerito } \\
\text { barbiescamado }\end{array}$ & & & \\
\hline
\end{tabular}




\begin{tabular}{|c|c|c|c|c|c|c|}
\hline Orden & Familia / Especie & Nombre de autor & Nombre común & $\begin{array}{l}\text { Amenaza } \\
\text { global } \\
\text { (IUCN) }\end{array}$ & $\begin{array}{c}\text { Amenaza } \\
\text { nacional } \\
\text { (Libro } \\
\text { rojo) }\end{array}$ & $\begin{array}{l}\text { Info. } \\
\text { de la } \\
\text { especie }\end{array}$ \\
\hline & $\begin{array}{l}\text { Myrmotherula } \\
\text { schisticolor }\end{array}$ & (Lawrence, 1865) & $\begin{array}{l}\text { Hormiguerito } \\
\text { pizarroso }\end{array}$ & & & \\
\hline & Formicivora grisea & (Boddaert, 1783) & $\begin{array}{l}\text { Hormiguerito } \\
\text { pechinegro }\end{array}$ & & & \\
\hline & $\begin{array}{l}\text { Drymophila } \\
\text { striaticeps }\end{array}$ & Chapman, 1912 & $\begin{array}{l}\text { Hormiguero } \\
\text { rabilargo }\end{array}$ & & & \\
\hline & $\begin{array}{l}\text { Cercomacroides } \\
\text { parkeri }\end{array}$ & G. R. Graves, 1997 & $\begin{array}{l}\text { Hormiguero de } \\
\text { parker }\end{array}$ & & & EN \\
\hline & Sipia palliata & (Todd, 1917) & $\begin{array}{l}\text { Hormiguero del } \\
\text { magdalena }\end{array}$ & NT & & \\
\hline & Hafferia immaculata & (Lafresnaye, 1845) & $\begin{array}{l}\text { Hormiguero } \\
\text { inmaculado }\end{array}$ & & & ML \\
\hline & Conopophagidae & & & & & \\
\hline & $\begin{array}{l}\text { Conopophaga } \\
\text { castaneiceps }\end{array}$ & P. L. Sclater, 1857 & $\begin{array}{l}\text { Zumbador } \\
\text { pechigrís }\end{array}$ & & & \\
\hline & Grallariidae & & & & & \\
\hline & Grallaria ruficapilla & Lafresnaye, 1842 & Tororoi comprapán & & & \\
\hline & Grallaria hypoleuca & P. L. Sclater, 1855 & $\begin{array}{l}\text { Tororoi } \\
\text { pechiblanco }\end{array}$ & & & \\
\hline & $\begin{array}{l}\text { Grallaricula } \\
\text { flavirostris }\end{array}$ & (P. L. Sclater, 1858) & $\begin{array}{l}\text { Tororoi } \\
\text { piquigualdo }\end{array}$ & NT & & \\
\hline & Rhinocryptidae & & & & & \\
\hline & Scytalopus atratus & Hellmayr, 1922 & $\begin{array}{l}\text { Tapaculo } \\
\text { cabeciblanco }\end{array}$ & & & \\
\hline & Scytalopus stilesi & $\begin{array}{l}\text { A. M. Cuervo, C. } \\
\text { D. Cadena, Krabbe } \\
\text { \& Renjifo, } 2005\end{array}$ & Tapaculo de stiles & & EN & EN \\
\hline & $\begin{array}{l}\text { Scytalopus } \\
\text { spillmanni }\end{array}$ & Stresemann, 1937 & $\begin{array}{l}\text { Tapaculo de } \\
\text { spillmann }\end{array}$ & & & \\
\hline & Formicariidae & & & & & \\
\hline & Formicarius analis & $\begin{array}{l}\text { (Orbigny \& } \\
\text { Lafresnaye, 1837) }\end{array}$ & Gallito carinegro & & & \\
\hline & $\begin{array}{l}\text { Formicarius } \\
\text { rufipectus* }\end{array}$ & Salvin, 1866 & Gallito pechirrufo & & & \\
\hline & Furnariidae & & & & & \\
\hline & Sclerurus mexicanus* & P. L. Sclater, 1857 & Tirahojas mexicano & & & \\
\hline & $\begin{array}{l}\text { Sittasomus } \\
\text { griseicapillus }\end{array}$ & (Vieillot, 1818) & $\begin{array}{l}\text { Trepatroncos } \\
\text { oliváceo }\end{array}$ & & & \\
\hline & $\begin{array}{l}\text { Dendrocincla } \\
\text { tyrannina }\end{array}$ & (Lafresnaye, 1851) & $\begin{array}{l}\text { Trepatroncos } \\
\text { cordillerano }\end{array}$ & & & \\
\hline
\end{tabular}




\begin{tabular}{|c|c|c|c|c|c|c|}
\hline Orden & Familia / Especie & Nombre de autor & Nombre común & $\begin{array}{c}\text { Amenaza } \\
\text { global } \\
\text { (IUCN) }\end{array}$ & $\begin{array}{c}\text { Amenaza } \\
\text { nacional } \\
\text { (Libro } \\
\text { rojo) }\end{array}$ & $\begin{array}{c}\text { Info. } \\
\text { de la } \\
\text { especie }\end{array}$ \\
\hline & $\begin{array}{l}\text { Dendrocincla } \\
\text { fuliginosa }\end{array}$ & (Vieillot, 1818) & $\begin{array}{l}\text { Trepatroncos } \\
\text { pardo }\end{array}$ & & & \\
\hline & $\begin{array}{l}\text { Glyphorynchus } \\
\text { spirurus }\end{array}$ & (Vieillot, 1819) & $\begin{array}{l}\text { Trepatroncos } \\
\text { pico de uña }\end{array}$ & & & \\
\hline & $\begin{array}{l}\text { Xiphocolaptes } \\
\text { promeropirhynchus }\end{array}$ & (Lesson, 1840) & $\begin{array}{l}\text { Trepatroncos } \\
\text { gigante }\end{array}$ & & & \\
\hline & $\begin{array}{l}\text { Xiphorhynchus } \\
\text { erythropygius }\end{array}$ & (P. L. Sclater, 1860) & $\begin{array}{l}\text { Trepatroncos } \\
\text { manchado }\end{array}$ & & & \\
\hline & $\begin{array}{l}\text { Campylorhamphus } \\
\text { trochilirostris }\end{array}$ & (Lichtenstein, 1820) & Guadañero rojizo & & & \\
\hline & $\begin{array}{l}\text { Campylorhamphus } \\
\text { pusillus }\end{array}$ & (P. L. Sclater, 1860) & $\begin{array}{l}\text { Guadañero } \\
\text { estriado }\end{array}$ & & & ML \\
\hline & $\begin{array}{l}\text { Xiphorhynchus } \\
\text { triangularis }\end{array}$ & (Lafresnaye, 1842) & $\begin{array}{l}\text { Trepatroncos } \\
\text { perlado }\end{array}$ & & & \\
\hline & $\begin{array}{l}\text { Lepidocolaptes } \\
\text { souleyetii }\end{array}$ & (Des Murs, 1849) & $\begin{array}{l}\text { Trepatroncos } \\
\text { campestre }\end{array}$ & & & \\
\hline & $\begin{array}{l}\text { Lepidocolaptes } \\
\text { lacrymiger }\end{array}$ & (Des Murs, 1849) & $\begin{array}{l}\text { Trepatroncos } \\
\text { montañero }\end{array}$ & & & \\
\hline & Xenops minutus & (Sparrman, 1788) & Xenops pardusco & & & \\
\hline & Xenops rutilans & Temminck, 1821 & Xenops estriado & & & \\
\hline & $\begin{array}{l}\text { Premnornis } \\
\text { guttuliger }\end{array}$ & (Sclater, PL, 1864) & $\begin{array}{l}\text { Corretroncos } \\
\text { alirrufo }\end{array}$ & & & \\
\hline & $\begin{array}{l}\text { Anabacerthia } \\
\text { striaticollis }\end{array}$ & Lafresnaye, 1841 & $\begin{array}{l}\text { Hojarasquero } \\
\text { montañero }\end{array}$ & & & \\
\hline & Dendroma rufa & (Vieillot, 1818) & $\begin{array}{l}\text { Hojarasquero } \\
\text { ocráceo }\end{array}$ & & & \\
\hline & $\begin{array}{l}\text { Automolus } \\
\text { ochrolaemus }\end{array}$ & (Tschudi, 1844) & $\begin{array}{l}\text { Hojarasquero } \\
\text { oliváceo }\end{array}$ & & & \\
\hline & $\begin{array}{l}\text { Premnoplex } \\
\text { brunnescens }\end{array}$ & (P. L. Sclater, 1856) & $\begin{array}{l}\text { Corretroncos } \\
\text { barranquero }\end{array}$ & & & \\
\hline & $\begin{array}{l}\text { Cranioleuca } \\
\text { erythrops }\end{array}$ & (P. L. Sclater, 1860) & $\begin{array}{l}\text { Rastrojero } \\
\text { rubicundo }\end{array}$ & & & \\
\hline & Synallaxis brachyura & Lafresnaye, 1843 & Chamicero pizarra & & & \\
\hline & Synallaxis albescens & Temminck, 1823 & Chamicero pálido & & & \\
\hline & Synallaxis azarae & Orbigny, 1835 & Chamicero piscuís & & & \\
\hline & \multicolumn{6}{|l|}{ Pipridae } \\
\hline & Masius chrysopterus & (Lafresnaye, 1843) & Saltarín moñudo & & & \\
\hline & Corapipo leucorrhoa & (P. L. Sclater, 1863) & $\begin{array}{l}\text { Saltarín } \\
\text { gorgiblanco }\end{array}$ & & & \\
\hline
\end{tabular}




\begin{tabular}{|c|c|c|c|c|c|c|}
\hline Orden & Familia / Especie & Nombre de autor & Nombre común & $\begin{array}{c}\text { Amenaza } \\
\text { global } \\
\text { (IUCN) }\end{array}$ & $\begin{array}{c}\text { Amenaza } \\
\text { nacional } \\
\text { (Libro } \\
\text { rojo) }\end{array}$ & $\begin{array}{c}\text { Info. } \\
\text { de la } \\
\text { especie }\end{array}$ \\
\hline & Lepidothrix coronata & (Spix, 1825) & Saltarín coroniazul & & & \\
\hline & Manacus manacus & (Linnaeus, 1766) & $\begin{array}{l}\text { Saltarín } \\
\text { barbiblanco }\end{array}$ & & & \\
\hline & $\begin{array}{l}\text { Machaeropterus } \\
\text { striolatus }\end{array}$ & (Hahn, 1819) & Saltarín rayado & & & \\
\hline & Dixiphia pipra & (Linnaeus, 1758) & $\begin{array}{l}\text { Saltarín } \\
\text { cabeciblanco }\end{array}$ & & & \\
\hline & \multicolumn{6}{|l|}{ Cotingidae } \\
\hline & Pipreola riefferii & $\begin{array}{l}\text { (Boissonneau, } \\
\text { 1840) }\end{array}$ & Frutero verdinegro & & & \\
\hline & Pipreola aureopectus* & (Lafresnaye, 1843) & $\begin{array}{l}\text { Frutero } \\
\text { pechidorado }\end{array}$ & & & \\
\hline & Ampelioides tschudii* & (G. R. Gray, 1846) & Frutero escamado & & & \\
\hline & Rupicola peruvianus & (Latham, 1790) & Gallito de las rocas & & & \\
\hline & Querula purpurata & $\begin{array}{l}\text { (Statius Muller, } \\
1776)\end{array}$ & Cuaba & & & \\
\hline & \multicolumn{6}{|l|}{ Tityridae } \\
\hline & Tityra inquisitor & (Lichtenstein, 1823) & Titira capirotada & & & \\
\hline & Tityra semifasciata & (Spix, 1825) & Titira enmascarada & & & \\
\hline & $\begin{array}{l}\text { Pachyramphus } \\
\text { versicolor }\end{array}$ & (Hartlaub, 1843) & Cabezón barrado & & & \\
\hline & $\begin{array}{l}\text { Pachyramphus } \\
\text { cinnamomeus }\end{array}$ & Lawrence, 1861 & Cabezón canelo & & & \\
\hline & $\begin{array}{l}\text { Pachyramphus } \\
\text { polychopterus }\end{array}$ & (Vieillot, 1818) & Cabezón aliblanco & & & \\
\hline & \multicolumn{6}{|l|}{ Onychorhynchidae } \\
\hline & $\begin{array}{l}\text { Onychorhynchus } \\
\text { coronatus }\end{array}$ & $\begin{array}{l}\text { (Statius Muller, } \\
1776)\end{array}$ & Atrapamoscas real & & & \\
\hline & Myiobius atricaudus & Lawrence, 1863 & $\begin{array}{l}\text { Atrapamoscas } \\
\text { colinegro }\end{array}$ & & & \\
\hline & \multicolumn{6}{|l|}{ Tyrannidae } \\
\hline & $\begin{array}{l}\text { Platyrinchus } \\
\text { mystaceus }\end{array}$ & Vieillot, 1818 & $\begin{array}{l}\text { Pico de pala } \\
\text { crestiamarillo }\end{array}$ & & & \\
\hline & $\begin{array}{l}\text { Pseudotriccus } \\
\text { pelzelni* }\end{array}$ & $\begin{array}{l}\text { Taczanowski \& } \\
\text { Berlepsch, } 1885\end{array}$ & $\begin{array}{l}\text { Tiranuelo } \\
\text { broncioliva }\end{array}$ & & & \\
\hline & $\begin{array}{l}\text { Phylloscartes } \\
\text { poecilotis }\end{array}$ & (P. L. Sclater, 1862) & $\begin{array}{l}\text { Atrapamoscas } \\
\text { variegado }\end{array}$ & & & \\
\hline
\end{tabular}




\begin{tabular}{|c|c|c|c|c|c|c|}
\hline Orden & Familia / Especie & Nombre de autor & Nombre común & $\begin{array}{c}\text { Amenaza } \\
\text { global } \\
\text { (IUCN) }\end{array}$ & $\begin{array}{c}\text { Amenaza } \\
\text { nacional } \\
\text { (Libro } \\
\text { rojo) }\end{array}$ & $\begin{array}{c}\text { Info. } \\
\text { de la } \\
\text { especie }\end{array}$ \\
\hline & $\begin{array}{l}\text { Phylloscartes } \\
\text { ophthalmicus }\end{array}$ & $\begin{array}{l}\text { (Taczanowski, } \\
1874 \text { ) }\end{array}$ & $\begin{array}{l}\text { Atrapamoscas } \\
\text { marmóreo }\end{array}$ & & & \\
\hline & $\begin{array}{l}\text { Mionectes } \\
\text { striaticollis }\end{array}$ & $\begin{array}{l}\text { (Orbigny \& } \\
\text { Lafresnaye, 1837) }\end{array}$ & $\begin{array}{l}\text { Atrapamoscas } \\
\text { estriado }\end{array}$ & & & \\
\hline & Mionectes olivaceus & Lawrence, 1868 & $\begin{array}{l}\text { Atrapamoscas } \\
\text { olivaceo }\end{array}$ & & & \\
\hline & Mionectes oleagineus & (Lichtenstein, 1823) & $\begin{array}{l}\text { Atrapamoscas } \\
\text { ocráceo }\end{array}$ & & & \\
\hline & $\begin{array}{l}\text { Leptopogon } \\
\text { superciliaris }\end{array}$ & Tschudi, 1844 & $\begin{array}{l}\text { Atrapamoscas } \\
\text { sepia }\end{array}$ & & & \\
\hline & Lophotriccus pileatus & (Tschudi, 1844) & $\begin{array}{l}\text { Tiranuelo } \\
\text { crestibarrado }\end{array}$ & & & \\
\hline & $\begin{array}{l}\text { Todirostrum } \\
\text { cinereum }\end{array}$ & (Linnaeus, 1766) & Espatulilla común & & & \\
\hline & Myiotriccus ornatus & (Lafresnaye, 1853) & $\begin{array}{l}\text { Atrapamoscas } \\
\text { ornado }\end{array}$ & & & \\
\hline & $\begin{array}{l}\text { Pyrrhomyias } \\
\text { cinnamomeus }\end{array}$ & $\begin{array}{l}\text { (Orbigny \& } \\
\text { Lafresnaye, 1837) }\end{array}$ & $\begin{array}{l}\text { Atrapamoscas } \\
\text { canela }\end{array}$ & & & \\
\hline & Zimmerius chrysops & (P. L. Sclater, 1859) & $\begin{array}{l}\text { Tiranuelo } \\
\text { cejiamarillo }\end{array}$ & & & \\
\hline & $\begin{array}{l}\text { Camptostoma } \\
\text { obsoletum }\end{array}$ & (Temminck, 1824) & Tiranuelo silbador & & & \\
\hline & Elaenia flavogaster & (Thunberg, 1822) & Elaenia copetona & & & \\
\hline & Elaenia frantzii & Lawrence, 1865 & Elaenia montañera & & & ML \\
\hline & Phaeomyias murina & (Spix, 1825) & Tiranuelo murino & & & \\
\hline & Serpophaga cinerea & (Tschudi, 1844) & $\begin{array}{l}\text { Tiranuelo salta } \\
\text { arroyo }\end{array}$ & & & \\
\hline & Legatus leucophaius & (Vieillot, 1818) & $\begin{array}{l}\text { Atrapamoscas } \\
\text { pirata }\end{array}$ & & & \\
\hline & Pitangus sulphuratus & (Linnaeus, 1766) & Bichofué & & & \\
\hline & Pitangus lictor & (Lichtenstein, 1823) & Bichofué chico & & & \\
\hline & $\begin{array}{l}\text { Myiodynastes } \\
\text { chrysocephalus }\end{array}$ & (Tschudi, 1844) & $\begin{array}{l}\text { Atrapamoscas } \\
\text { lagartero }\end{array}$ & & & \\
\hline & $\begin{array}{l}\text { Myiodynastes } \\
\text { maculatus }\end{array}$ & $\begin{array}{l}\text { (Statius Muller, } \\
1776)\end{array}$ & Sirirí rayado & & & \\
\hline & $\begin{array}{l}\text { Myiozetetes } \\
\text { cayanensis }\end{array}$ & (Linnaeus, 1766) & Suelda crestinegra & & & \\
\hline & Myiozetetes similis & (Spix, 1825) & Suelda social & & & \\
\hline & Conopias cinchoneti & (Tschudi, 1844) & Suelda cejiamarilla & VU & & \\
\hline
\end{tabular}




\begin{tabular}{|c|c|c|c|c|c|c|}
\hline Orden & Familia / Especie & Nombre de autor & Nombre común & $\begin{array}{l}\text { Amenaza } \\
\text { global } \\
\text { (IUCN) }\end{array}$ & $\begin{array}{c}\text { Amenaza } \\
\text { nacional } \\
\text { (Libro } \\
\text { rojo) }\end{array}$ & $\begin{array}{l}\text { Info. } \\
\text { de la } \\
\text { especie }\end{array}$ \\
\hline & $\begin{array}{l}\text { Tyrannus } \\
\text { melancholicus }\end{array}$ & Vieillot, 1819 & Sirirí común & & & \\
\hline & Tyrannus savana & Vieillot, 1808 & Sirirí tijeretón & & & $\mathrm{MBr}$ \\
\hline & $\begin{array}{l}\text { Myiarchus } \\
\text { tuberculifer }\end{array}$ & $\begin{array}{l}\text { (Orbigny \& } \\
\text { Lafresnaye, 1837) }\end{array}$ & $\begin{array}{l}\text { Atrapamoscas } \\
\text { cabecinegro }\end{array}$ & & & \\
\hline & Myiarchus cephalotes & Taczanowski, 1880 & $\begin{array}{l}\text { Atrapamoscas } \\
\text { montañero }\end{array}$ & & & \\
\hline & Colonia colonus & (Vieillot, 1818) & $\begin{array}{l}\text { Atrapamoscas } \\
\text { rabijunco }\end{array}$ & & & \\
\hline & Myiophobus fasciatus & $\begin{array}{l}\text { (Statius Muller, } \\
1776 \text { ) }\end{array}$ & $\begin{array}{l}\text { Atrapamoscas } \\
\text { pechirrayado }\end{array}$ & & & \\
\hline & $\begin{array}{l}\text { Ochthoeca } \\
\text { cinnamomeiventris }\end{array}$ & (Lafresnaye, 1843) & Pitajo torrentero & & & \\
\hline & $\begin{array}{l}\text { Knipolegus } \\
\text { poecilurus* }\end{array}$ & (P. L. Sclater, 1862) & $\begin{array}{l}\text { Atrapamoscas } \\
\text { renegrido }\end{array}$ & & & \\
\hline & $\begin{array}{l}\text { Myiotheretes } \\
\text { striaticollis }\end{array}$ & (P. L. Sclater, 1853) & $\begin{array}{l}\text { Atrapamoscas } \\
\text { chiflaperros }\end{array}$ & & & \\
\hline & Sayornis nigricans & (Swainson, 1827) & $\begin{array}{l}\text { Atrapamoscas } \\
\text { cuidapuentes }\end{array}$ & & & \\
\hline & Empidonax virescens & (Vieillot, 1818) & $\begin{array}{l}\text { Atrapamoscas } \\
\text { verdoso }\end{array}$ & & & $\mathrm{MB}$ \\
\hline & Empidonax traillii & (Audubon, 1828) & $\begin{array}{l}\text { Atrapamoscas } \\
\text { saucero }\end{array}$ & & & $\mathrm{MB}$ \\
\hline & Contopus cooperi & (Nuttall, 1831) & Pibí boreal & NT & NT & MB \\
\hline & Contopus fumigatus & $\begin{array}{l}\text { (Orbigny \& } \\
\text { Lafresnaye, 1837) }\end{array}$ & Pibi oscuro & & & \\
\hline & Contopus virens & (Linnaeus, 1766) & Pibí oriental & & & MB \\
\hline & Contopus cinereus & (Spix, 1825) & Pibí tropical & & & \\
\hline & Vireonidae & & & & & \\
\hline & Cyclarhis nigrirostris & Lafresnaye, 1842 & $\begin{array}{l}\text { Verderón } \\
\text { piquinegro }\end{array}$ & & & \\
\hline & $\begin{array}{l}\text { Pachysylvia } \\
\text { semibrunnea }\end{array}$ & (Lafresnaye, 1845) & Verderón castaño & & & \\
\hline & Vireo leucophrys & (Lafresnaye, 1844) & $\begin{array}{l}\text { Verderón } \\
\text { montañero }\end{array}$ & & & \\
\hline & Corvidae & & & & & \\
\hline & Cyanocorax affinis & Pelzeln, 1856 & $\begin{array}{l}\text { Carriquí pechi- } \\
\text { blanco }\end{array}$ & & & \\
\hline & Cyanocorax yncas & (Boddaert, 1783) & $\begin{array}{l}\text { Carriquí de } \\
\text { montaña }\end{array}$ & & & \\
\hline
\end{tabular}




\begin{tabular}{|c|c|c|c|c|c|c|}
\hline Orden & Familia / Especie & Nombre de autor & Nombre común & $\begin{array}{c}\text { Amenaza } \\
\text { global } \\
\text { (IUCN) }\end{array}$ & $\begin{array}{c}\text { Amenaza } \\
\text { nacional } \\
\text { (Libro } \\
\text { rojo) }\end{array}$ & $\begin{array}{c}\text { Info. } \\
\text { de la } \\
\text { especie }\end{array}$ \\
\hline & \multicolumn{6}{|l|}{ Hirundinidae } \\
\hline & $\begin{array}{l}\text { Pygochelidon } \\
\text { cyanoleuca }\end{array}$ & (Vieillot, 1817) & $\begin{array}{l}\text { Golondrina azul y } \\
\text { blanca }\end{array}$ & & & \\
\hline & Atticora tibialis & (Cassin, 1853) & $\begin{array}{l}\text { Golondrina } \\
\text { selvática }\end{array}$ & & & \\
\hline & $\begin{array}{l}\text { Stelgidopteryx } \\
\text { ruficollis }\end{array}$ & (Vieillot, 1817) & $\begin{array}{l}\text { Golondrina } \\
\text { barranquera }\end{array}$ & & & \\
\hline & Hirundo rustica & Linnaeus, 1758 & Golondrina tijereta & & & MB \\
\hline & \multicolumn{6}{|l|}{ Troglodytidae } \\
\hline & $\begin{array}{l}\text { Microcerculus } \\
\text { marginatus }\end{array}$ & (P. L. Sclater, 1855) & $\begin{array}{l}\text { Cucarachero } \\
\text { ruiseñor }\end{array}$ & & & \\
\hline & Troglodytes aedon & Vieillot, 1809 & $\begin{array}{l}\text { Cucarachero } \\
\text { común }\end{array}$ & & & \\
\hline & $\begin{array}{l}\text { Campylorhynchus } \\
\text { zonatus }\end{array}$ & (Lesson, 1832) & $\begin{array}{l}\text { Cucarachero } \\
\text { matraquero }\end{array}$ & & & \\
\hline & Pheugopedius spadix & Bangs, 1910 & $\begin{array}{l}\text { Cucarachero } \\
\text { cabecinegro }\end{array}$ & & & \\
\hline & $\begin{array}{l}\text { Pheugopedius } \\
\text { fasciatoventris }\end{array}$ & (Lafresnaye, 1845) & $\begin{array}{l}\text { Cucarachero } \\
\text { buchinegro }\end{array}$ & & & \\
\hline & $\begin{array}{l}\text { Cantorchilus } \\
\text { nigricapillus }\end{array}$ & (P. L. Sclater, 1860) & $\begin{array}{l}\text { Cacarachero } \\
\text { ribereño }\end{array}$ & & & \\
\hline & $\begin{array}{l}\text { Cinnycerthia } \\
\text { olivascens }\end{array}$ & Sharpe, 1882 & Cucarachero sepia & & & \\
\hline & $\begin{array}{l}\text { Henicorhina } \\
\text { leucosticta }\end{array}$ & (Cabanis, 1847) & $\begin{array}{l}\text { Cucarachero } \\
\text { pechiblanco }\end{array}$ & & & \\
\hline & $\begin{array}{l}\text { Henicorhina } \\
\text { leucophrys }\end{array}$ & (Tschudi, 1844) & $\begin{array}{l}\text { Cucarachero } \\
\text { pechigrís }\end{array}$ & & & \\
\hline & \multicolumn{6}{|l|}{ Cinclidae } \\
\hline & $\begin{array}{l}\text { Cinclus } \\
\text { leucocephalus }\end{array}$ & Tschudi, 1844 & Mirlo acuático & & & \\
\hline & \multicolumn{6}{|l|}{ Turdidae } \\
\hline & Myadestes ralloides & (Orbigny, 1840) & Solitario andino & & & \\
\hline & Catharus ustulatus & (Nuttall, 1840) & Zorzal buchipecoso & & & $\mathrm{MB}$ \\
\hline & Turdus leucops & Taczanowski, 1877 & Mirla ojiblanca & & & \\
\hline & Turdus leucomelas & Vieillot, 1818 & Mirla cabecigrís & & & \\
\hline & Turdus ignobilis & P. L. Sclater, 1858 & Mirla embarradora & & & \\
\hline & Turdus fuscater & $\begin{array}{l}\text { Orbigny \& } \\
\text { Lafresnaye, } 1837\end{array}$ & Mirla patinaranja & & & \\
\hline
\end{tabular}




\begin{tabular}{|c|c|c|c|c|c|c|}
\hline Orden & Familia / Especie & Nombre de autor & Nombre común & $\begin{array}{c}\text { Amenaza } \\
\text { global } \\
\text { (IUCN) }\end{array}$ & $\begin{array}{c}\text { Amenaza } \\
\text { nacional } \\
\text { (Libro } \\
\text { rojo) }\end{array}$ & $\begin{array}{c}\text { Info. } \\
\text { de la } \\
\text { especie }\end{array}$ \\
\hline & Turdus serranus & Tschudi, 1844 & Mirla serrana & & & \\
\hline & \multicolumn{6}{|l|}{ Mimidae } \\
\hline & Mimus gilvus & (Vieillot, 1808) & Sinsonte común & & & \\
\hline & \multicolumn{6}{|l|}{ Fringillidae } \\
\hline & $\begin{array}{l}\text { Spinus } \\
\text { xanthogastrus }\end{array}$ & $\begin{array}{l}\text { (Du Bus de } \\
\text { Gisignies, 1855) }\end{array}$ & $\begin{array}{l}\text { Jilguero } \\
\text { pechinegro }\end{array}$ & & & \\
\hline & Spinus psaltria & (Say, 1822) & Jilguero aliblanco & & & \\
\hline & Euphonia laniirostris & $\begin{array}{l}\text { d'Orbigny \& } \\
\text { Lafresnaye, } 1837\end{array}$ & $\begin{array}{l}\text { Eufonia } \\
\text { gorgiamarilla }\end{array}$ & & & \\
\hline & $\begin{array}{l}\text { Euphonia } \\
\text { cyanocephala }\end{array}$ & (Vieillot, 1819) & Eufonia cabeciazul & & & \\
\hline & $\begin{array}{l}\text { Euphonia } \\
\text { xanthogaster }\end{array}$ & Sundevall, 1834 & Eufonia común & & & \\
\hline & Chlorophonia cyanea & (Thunberg, 1822) & $\begin{array}{l}\text { Clorofonia } \\
\text { verdiazul }\end{array}$ & & & \\
\hline & $\begin{array}{l}\text { Chlorophonia } \\
\text { pyrrhophrys }\end{array}$ & (P. L. Sclater, 1851) & Eufonia ferrugínea & & & \\
\hline & \multicolumn{6}{|l|}{ Passerellidae } \\
\hline & $\begin{array}{l}\text { Chlorospingus } \\
\text { flavigularis }\end{array}$ & (P. L. Sclater, 1852) & $\begin{array}{l}\text { Montero } \\
\text { gorgiamarillo }\end{array}$ & & & \\
\hline & $\begin{array}{l}\text { Chlorospingus } \\
\text { flavopectus }\end{array}$ & (Lafresnaye, 1840) & Montero ojiblanco & & & \\
\hline & Arremon atricapillus & (Lawrence, 1874) & $\begin{array}{l}\text { Gorrión montés } \\
\text { cabecinegro }\end{array}$ & & & \\
\hline & $\begin{array}{l}\text { Arremon } \\
\text { aurantiirostris }\end{array}$ & Lafresnaye, 1847 & $\begin{array}{l}\text { Pinzón } \\
\text { piquidorado }\end{array}$ & & & \\
\hline & $\begin{array}{l}\text { Arremon } \\
\text { brunneinucha }\end{array}$ & (Lafresnaye, 1839) & $\begin{array}{l}\text { Gorrión montés } \\
\text { collarejo }\end{array}$ & & & \\
\hline & $\begin{array}{l}\text { Arremon } \\
\text { castaneiceps* }\end{array}$ & (P. L. Sclater, 1860) & Pinzón oliva & NT & & \\
\hline & Zonotrichia capensis & $\begin{array}{l}\text { (P. L. Statius Mü- } \\
\text { ller, 1776) }\end{array}$ & Copetón común & & & \\
\hline & Atlapetes schistaceus & $\begin{array}{l}\text { (Boissonneau, } \\
\text { 1840) }\end{array}$ & $\begin{array}{l}\text { Gorrión montés } \\
\text { pizarra }\end{array}$ & & & \\
\hline & Atlapetes albinucha & $\begin{array}{l}\text { (Lafresnaye \& } \\
\text { d'Orbigny, 1838) }\end{array}$ & $\begin{array}{l}\text { Gorrión montés } \\
\text { gorgiamarillo }\end{array}$ & & & \\
\hline & Atlapetes flaviceps & Chapman, 1912 & $\begin{array}{l}\text { Gorrión montés de } \\
\text { anteojos }\end{array}$ & $\mathrm{EN}$ & VU & EN \\
\hline & $\begin{array}{l}\text { Atlapetes } \\
\text { pallidinucha }\end{array}$ & $\begin{array}{l}\text { (Boissonneau, } \\
1840 \text { ) }\end{array}$ & $\begin{array}{l}\text { Gorrión montés } \\
\text { cabeciblanco }\end{array}$ & & & \\
\hline
\end{tabular}




\begin{tabular}{|c|c|c|c|c|c|c|}
\hline Orden & Familia / Especie & Nombre de autor & Nombre común & $\begin{array}{c}\text { Amenaza } \\
\text { global } \\
\text { (IUCN) }\end{array}$ & $\begin{array}{c}\text { Amenaza } \\
\text { nacional } \\
\text { (Libro } \\
\text { rojo) }\end{array}$ & $\begin{array}{l}\text { Info. } \\
\text { de la } \\
\text { especie }\end{array}$ \\
\hline & Atlapetes latinuchus & $\begin{array}{l}\text { (Du Bus de Gisig- } \\
\text { nies, 1855) }\end{array}$ & $\begin{array}{l}\text { Gorrión montés } \\
\text { cabecirrufo }\end{array}$ & & & \\
\hline & \multicolumn{6}{|l|}{ Icteridae } \\
\hline & $\begin{array}{l}\text { Psarocolius } \\
\text { angustifrons }\end{array}$ & (Spix, 1824) & $\begin{array}{l}\text { Oropéndola } \\
\text { variable }\end{array}$ & & & \\
\hline & $\begin{array}{l}\text { Psarocolius } \\
\text { decumanus }\end{array}$ & (Pallas, 1769) & $\begin{array}{l}\text { Oropéndola } \\
\text { crestada }\end{array}$ & & & \\
\hline & Cacicus uropygialis & (Lafresnaye, 1843) & Arrendajo culirrojo & & & \\
\hline & Cacicus chrysonotus & $\begin{array}{l}\text { (Lafresnaye \& Or- } \\
\text { bigny, 1838) }\end{array}$ & $\begin{array}{l}\text { Arrendajo } \\
\text { montañero }\end{array}$ & & & \\
\hline & Icterus auricapillus & Cassin, 1848 & Turpial cabecirrojo & & & \\
\hline & Icterus chrysater & (Lesson, 1844) & Turpial montañero & & & \\
\hline & Icterus galbula & (Linnaeus, 1758) & $\begin{array}{l}\text { Turpial de } \\
\text { baltimore }\end{array}$ & & & $\mathrm{MB}$ \\
\hline & $\begin{array}{l}\text { Molothrus } \\
\text { oryzivorus }\end{array}$ & (Gmelin, 1788) & Chamón gigante & & & \\
\hline & $\begin{array}{l}\text { Molothrus } \\
\text { bonariensis }\end{array}$ & (Gmelin, 1789) & Chamón parásito & & & \\
\hline & $\begin{array}{l}\text { Hypopyrrhus } \\
\text { pyrohypogaster }\end{array}$ & (Tarragon, 1847) & Cacique candela & VU & VU & EN \\
\hline
\end{tabular}

\section{Parulidae}

\begin{tabular}{|c|c|c|c|}
\hline $\begin{array}{l}\text { Parkesia } \\
\text { noveboracensis }\end{array}$ & (J. F. Gmelin, 1789) & Reinita acuática & MB \\
\hline $\begin{array}{l}\text { Vermivora } \\
\text { chrysoptera }\end{array}$ & (Linnaeus, 1766) & Reinita alidorada & MB \\
\hline Mniotilta varia & (Linnaeus, 1766) & Cebrita trepadora & MB \\
\hline Protonotaria citrea & (Boddaert, 1783) & $\begin{array}{l}\text { Reinita } \\
\text { cabecidorada }\end{array}$ & $\mathrm{MBr}$ \\
\hline Leiothlypis peregrina & (A. Wilson, 1811) & Reinita verderona & $\mathrm{MB}$ \\
\hline Oporornis agilis & (A. Wilson, 1812) & Reinita pechigrís & MB \\
\hline $\begin{array}{l}\text { Geothlypis } \\
\text { philadelphia }\end{array}$ & (A. Wilson, 1810) & Reinita enlutada & MB \\
\hline Setophaga ruticilla & (Linnaeus, 1758) & Reinita norteña & MB \\
\hline Setophaga pitiayumi & (Vieillot, 1817) & Reinita tropical & ML \\
\hline Setophaga castanea & (A. Wilson, 1810) & Reinita castaña & $\mathrm{MB}$ \\
\hline Setophaga fusca & $\begin{array}{l}\text { (Statius Muller, } \\
1776)\end{array}$ & $\begin{array}{l}\text { Reinita } \\
\text { gorginaranja }\end{array}$ & $\mathrm{MB}$ \\
\hline Setophaga petechia & (Linnaeus, 1766) & Reinita dorada & $\mathrm{MBr}$ \\
\hline
\end{tabular}




\begin{tabular}{|c|c|c|c|c|c|c|}
\hline Orden & Familia / Especie & Nombre de autor & Nombre común & $\begin{array}{l}\text { Amenaza } \\
\text { global } \\
\text { (IUCN) }\end{array}$ & $\begin{array}{c}\text { Amenaza } \\
\text { nacional } \\
\text { (Libro } \\
\text { rojo) }\end{array}$ & $\begin{array}{l}\text { Info. } \\
\text { de la } \\
\text { especie }\end{array}$ \\
\hline & Setophaga striata & (J. R. Forster, 1772) & Reinita rayada & & & $\mathrm{MB}$ \\
\hline & $\begin{array}{l}\text { Myiothlypis } \\
\text { luteoviridis }\end{array}$ & (Bonaparte, 1845) & Arañero cetrino & & & \\
\hline & $\begin{array}{l}\text { Myiothlypis } \\
\text { fulvicauda }\end{array}$ & (von Spix, 1825) & Arañero ribereño & & & \\
\hline & Myiothlypis coronata & (Tschudi, 1844) & Arañero coronado & & & \\
\hline & Basileuterus rufifrons & (Swainson, 1838) & $\begin{array}{l}\text { Arañero } \\
\text { cabecirrufo }\end{array}$ & & & \\
\hline & $\begin{array}{l}\text { Basileuterus } \\
\text { tristriatus }\end{array}$ & (Tschudi, 1844) & $\begin{array}{l}\text { Arañero } \\
\text { cabecirrayado }\end{array}$ & & & \\
\hline & Cardellina canadensis & (Linnaeus, 1766) & Reinita de canadá & & & MB \\
\hline & Myioborus miniatus & (Swainson, 1827) & $\begin{array}{l}\text { Abanico } \\
\text { pechinegro }\end{array}$ & & & \\
\hline & Mitrospingidae & & & & & \\
\hline & Mitrospingus cassinii & (Lawrence, 1861) & $\begin{array}{l}\text { Maraquera } \\
\text { carisucia }\end{array}$ & & & \\
\hline & Cardinalidae & & & & & \\
\hline & Piranga flava & (Vieillot, 1822) & Piranga bermeja & & & ML \\
\hline & Piranga rubra & (Linnaeus, 1758) & Piranga abejera & & & $\mathrm{MBr}$ \\
\hline & Piranga olivacea & (J. F. Gmelin, 1789) & Piranga alinegra & & & MB \\
\hline & Piranga leucoptera & Trudeau, 1840 & Piranga aliblanca & & & \\
\hline & Habia gutturalis & (P. L. Sclater, 1854) & Habia ceniza & NT & & $\mathrm{EN}$ \\
\hline & Habia cristata & (Lawrence, 1875) & Habia Copetona & & & EN \\
\hline & $\begin{array}{l}\text { Pheucticus } \\
\text { ludovicianus }\end{array}$ & (Linnaeus, 1766) & $\begin{array}{l}\text { Picogordo } \\
\text { degollado }\end{array}$ & & & MB \\
\hline & Cyanoloxia cyanoides & (Lafresnaye, 1847) & Azulón silvícola & & & \\
\hline & Thraupidae & & & & & \\
\hline & $\begin{array}{l}\text { Sericossypha } \\
\text { albocristata }\end{array}$ & (Lafresnaye, 1843) & Pollo de monte & VU & & \\
\hline & Chlorophanes spiza & (Linnaeus, 1758) & Mielero verde & & & \\
\hline & $\begin{array}{l}\text { Heterospingus } \\
\text { xanthopygius }\end{array}$ & (P. L. Sclater, 1855) & $\begin{array}{l}\text { Chambergo } \\
\text { cejirrojo }\end{array}$ & & & \\
\hline & Hemithraupis güira & (Linnaeus, 1766) & Pintasilgo güira & & & \\
\hline & $\begin{array}{l}\text { Hemithraupis } \\
\text { flavicollis }\end{array}$ & (Vieillot, 1818) & $\begin{array}{l}\text { Pintasilgo } \\
\text { culiamarillo }\end{array}$ & & & \\
\hline & Sicalis flaveola & (Linnaeus, 1766) & Canario coronado & & & \\
\hline
\end{tabular}




\begin{tabular}{|c|c|c|c|c|c|c|}
\hline Orden & Familia / Especie & Nombre de autor & Nombre común & $\begin{array}{l}\text { Amenaza } \\
\text { global } \\
\text { (IUCN) }\end{array}$ & $\begin{array}{c}\text { Amenaza } \\
\text { nacional } \\
\text { (Libro } \\
\text { rojo) }\end{array}$ & $\begin{array}{l}\text { Info. } \\
\text { de la } \\
\text { especie }\end{array}$ \\
\hline & Diglossa albilatera & Lafresnaye, 1843 & Picaflor aliblanco & & & \\
\hline & Diglossa caerulescens & (P. L. Sclater, 1856) & Picaflor azul & & & \\
\hline & Diglossa cyanea & (Lafresnaye, 1840) & Picaflor de antifaz & & & \\
\hline & Volatinia jacarina & (Linnaeus, 1766) & Espiguero saltarín & & & \\
\hline & Creurgops verticalis & P. L. Sclater, 1858 & Tangara crestirufa & & VU & \\
\hline & Loriotus luctuosus & $\begin{array}{l}\text { (d'Orbigny and } \\
\text { Lafresnaye, 1837) }\end{array}$ & Parlotero aliblanco & & & \\
\hline & Tachyphonus delatrii & Lafresnaye, 1847 & $\begin{array}{l}\text { Parlotero } \\
\text { occidental }\end{array}$ & & & \\
\hline & Tachyphonus rufus & (Boddaert, 1783) & $\begin{array}{l}\text { Parlotero } \\
\text { malcasado }\end{array}$ & & & \\
\hline & Eucometis penicillata & (von Spix, 1825) & Guicha hormiguera & & & \\
\hline & $\begin{array}{l}\text { Ramphocelus } \\
\text { dimidiatus }\end{array}$ & Lafresnaye, 1837 & Toche pico de plata & & & \\
\hline & $\begin{array}{l}\text { Ramphocelus } \\
\text { flammigerus }\end{array}$ & $\begin{array}{l}\text { (Jardine \& Selby, } \\
\text { 1833) }\end{array}$ & Toche enjalmado & & & \\
\hline & Cyanerpes caeruleus & (Linnaeus, 1758) & Mielero cerúleo & & & \\
\hline & Tersina viridis & (Illiger, 1811) & Azulejo golondrina & & & \\
\hline & Dacnis lineata & (J. F. Gmelin, 1789) & Dacnis carinegra & & & \\
\hline & Dacnis cayana & (Linnaeus, 1766) & Dacnis azul & & & ML \\
\hline & Sporophila minuta & (Linnaeus, 1758) & Espiguero ladrillo & & & \\
\hline & Sporophila funerea & (Sclater, pl, 1860) & $\begin{array}{l}\text { Arrocero } \\
\text { piquigrueso }\end{array}$ & & & \\
\hline & Sporophila angolensis & (Linnaeus, 1766) & $\begin{array}{l}\text { Arrocero } \\
\text { buchicastaño }\end{array}$ & & & \\
\hline & Sporophila luctuosa & (Lafresnaye, 1843) & $\begin{array}{l}\text { Espiguero } \\
\text { negriblanco }\end{array}$ & & & \\
\hline & Sporophila nigricollis & (Vieillot, 1823) & $\begin{array}{l}\text { Espiguero } \\
\text { capuchino }\end{array}$ & & & \\
\hline & Sporophila schistacea & (Lawrence, 1862) & Espiguero pizarra & & & \\
\hline & Saltator maximus & $\begin{array}{l}\text { (P. L. Statius Mü- } \\
\text { ller, 1776) }\end{array}$ & Saltator ajicero & & & \\
\hline & Saltator atripennis & P. L. Sclater, 1857 & Saltator alinegro & & & \\
\hline & Saltator striatipectus & Lafresnaye, 1847 & Saltator piojudío & & & \\
\hline & Saltator grossus* & (Linnaeus, 1766) & Piquigordo pizarra & & & \\
\hline & $\begin{array}{l}\text { Emberizoides } \\
\text { herbicola }\end{array}$ & (Vieillot, 1817) & Sabanero coludo & & & \\
\hline
\end{tabular}




\begin{tabular}{|c|c|c|c|c|c|c|}
\hline Orden & Familia / Especie & Nombre de autor & Nombre común & $\begin{array}{l}\text { Amenaza } \\
\text { global } \\
\text { (IUCN) }\end{array}$ & $\begin{array}{c}\text { Amenaza } \\
\text { nacional } \\
\text { (Libro } \\
\text { rojo) }\end{array}$ & $\begin{array}{l}\text { Info. } \\
\text { de la } \\
\text { especie }\end{array}$ \\
\hline & $\begin{array}{l}\text { Thlypopsis } \\
\text { superciliaris }\end{array}$ & (Lafresnaye, 1840) & $\begin{array}{l}\text { Hemispingus } \\
\text { cejiblanco }\end{array}$ & & & \\
\hline & Coereba flaveola & (Linnaeus, 1758) & Mielero común & & & \\
\hline & Tiaris olivaceus & (Linnaeus, 1766) & $\begin{array}{l}\text { Semillero } \\
\text { cariamarillo }\end{array}$ & & & \\
\hline & Melanospiza bicolor & (Linnaeus, 1766) & $\begin{array}{l}\text { Semillero } \\
\text { pechinegro }\end{array}$ & & & \\
\hline & $\begin{array}{l}\text { Chlorochrysa } \\
\text { nitidissima* }\end{array}$ & P. L. Sclater, 1874 & Tangara multicolor & VU & VU & EN \\
\hline & $\begin{array}{l}\text { Schistochlamys } \\
\text { melanopis }\end{array}$ & (Latham, 1790) & Pizarrita sabanera & & & \\
\hline & $\begin{array}{l}\text { Iridosornis } \\
\text { porphyrocephalus* }\end{array}$ & (P. L. Sclater, 1856) & $\begin{array}{l}\text { Musguerito } \\
\text { gargantilla }\end{array}$ & NT & & \\
\hline & Dubusia taeniata & $\begin{array}{l}\text { (Boissonneau, } \\
1840 \text { ) }\end{array}$ & Tangara diadema & & & \\
\hline & $\begin{array}{l}\text { Anisognathus } \\
\text { somptuosus }\end{array}$ & (Lesson, 1831) & Tangara primavera & & & \\
\hline & $\begin{array}{l}\text { Sporathraupis } \\
\text { cyanocephala }\end{array}$ & $\begin{array}{l}\text { d'Orbigny and La- } \\
\text { fresnaye, } 1837\end{array}$ & Azulejo montañero & & & \\
\hline & Stilpnia heinei & (Cabanis, 1850) & Tangara capirotada & & & \\
\hline & Stilpnia vitriolina & (Cabanis, 1851) & Tangara rastrojera & & & \\
\hline & Stilpnia larvata & $\begin{array}{l}\text { (du Bus de Gisig- } \\
\text { nies, 1846) }\end{array}$ & Tangara collareja & & & ML \\
\hline & Stilpnia cyanicollis & $\begin{array}{l}\text { (d'Orbigny \& La- } \\
\text { fresnaye, 1837) }\end{array}$ & Tangara real & & & \\
\hline & Tangara vassorii & $\begin{array}{l}\text { (Boissonneau, } \\
1840 \text { ) }\end{array}$ & Tangara negriazul & & & \\
\hline & Tangara nigroviridis & (Lafresnaye, 1843) & Tangara berilina & & & \\
\hline & Tangara labradorides & $\begin{array}{l}\text { (Boissonneau, } \\
1840 \text { ) }\end{array}$ & Tangara verdiplata & & & \\
\hline & Tangara inornata & (Gould, 1855) & Tangara cenicienta & & & \\
\hline & Tangara gyrola & (Linnaeus, 1758) & $\begin{array}{l}\text { Tangara } \\
\text { cabecirrufa }\end{array}$ & & & \\
\hline & $\begin{array}{l}\text { Tangara } \\
\text { xanthocephala }\end{array}$ & (Tschudi, 1844) & Tangara coronada & & & \\
\hline & Tangara arthus & Lesson, 1832 & Tangara dorada & & & \\
\hline & Thraupis episcopus & (Linnaeus, 1766) & Azulejo común & & & \\
\hline & Thraupis palmarum & $\begin{array}{l}\text { (zu Wied-Neu- } \\
\text { wied, 1821) }\end{array}$ & Azulejo palmero & & & \\
\hline & Ixothraupis guttata & (Cabanis, 1851) & Tangara pecosa & & & \\
\hline
\end{tabular}




\section{Daniela Gómez}

Sociedad Caldense de Ornitología

Manizales, Colombia

gomezdaniela006@gmail.com

https://orcid.org/0000-0003-0163-653X

\section{Kelly Orozco}

Universidad de Caldas

Manizales, Colombia

kellyjop@hotmail.com

https://orcid.org/0000-0002-2715-4443

Felipe Cardona

Sistema de Parques Nacionales Naturales de Colombia

Manizales, Colombia

andresfelipecardona.toro@gmail.com

https://orcid.org/0000-0003-3743-2272

Milton Pineda

Sistema de Parques Nacionales Naturales de Colombia

Manizales, Colombia

miorpi.1@gmail.com

https://orcid.org/0000-0003-4936-1790

Mary Luz Bedoya

Universidad de Caldas

Manizales, Colombia

maryluz.bedoya@ucaldas.edu.co

https://orcid.org/0000-0002-3162-4188

\section{David Ocampo}

Instituto de Investigación de Recursos Biológicos Alexander von Humboldt

Bogotá, Colombia

algorab2@gmail.com

https://orcid.org/0000-0003-1597-4038
Avifauna del Parque Nacional Natural Selva de Florencia (Samaná, Caldas, Colombia): nuevos registros y ampliaciones de distribución

Citación del artículo: : Gómez, D., Orozco, K., Cardona, F., Pineda, M., Bedoya, M.L. \& Ocampo, D. (2020). Avifauna del Parque Nacional Natural Selva de Florencia (Samaná, Caldas, Colombia): nuevos registros y ampliaciones de distribución. Biota colombiana, 21(2). 40-71. DOI: 10.21068/c2020.v21n02a03.

Recibido: 25 de septiembre de 2019

Aceptado: 4 de mayo 2020 